\title{
A constructive proof of the existence of Viterbi processes
}

\author{
Jüri Lember, Alexey Koloydenko
}

\begin{abstract}
Since the early days of digital communication, hidden Markov models (HMMs) have now been also routinely used in speech recognition, processing of natural languages, images, and in bioinformatics. In an HMM $\left(X_{i}, Y_{i}\right)_{i \geq 1}$, observations $X_{1}, X_{2}, \ldots$ are assumed to be conditionally independent given an "explanatory" Markov process $Y_{1}, Y_{2}, \ldots$, which itself is not observed; moreover, the conditional distribution of $X_{i}$ depends solely on $Y_{i}$. Central to the theory and applications of HMM is the Viterbi algorithm to find a maximum a posteriori (MAP) estimate $q_{1: n}=\left(q_{1}, q_{2}, \ldots, q_{n}\right)$ of $Y_{1: n}$ given observed data $x_{1: n}$. Maximum a posteriori paths are also known as Viterbi paths or alignments. Recently, attempts have been made to study the behavior of Viterbi alignments when $n \rightarrow \infty$. Thus, it has been shown that in some special cases a well-defined limiting Viterbi alignment exists. While innovative, these attempts have relied on rather strong assumptions and involved proofs which are existential. This work proves the existence of infinite Viterbi alignments in a more constructive manner and for a very general class of HMMs.
\end{abstract}

Index Terms-Asymptotic, HMM, maximum a posteriori path, Viterbi algorithm, Viterbi extraction, Viterbi training.

\section{INTRODUCTION}

$\mathbf{L}$ ET $Y=\left(Y_{i}\right)_{i \geq 1}$ be a Markov chain with state space $S=\{1, \ldots, K\}, K>1$, and transition matrix $\mathbb{P}=$ $\left(p_{i j}\right)_{i, j \in S}$. Suppose that $Y$ is irreducible and aperiodic, hence a unique stationary distribution $\pi=\pi \mathbb{P}$ exists; suppose further that $Y_{i} \sim \pi$ from time $i=1$. To every state $l \in S$, let us assign an emission distribution $P_{l}$ on $(\mathcal{X}, \mathcal{B})$, where $\mathcal{X}=\mathbb{R}^{D}$, the $D$ dimensional Euclidean space. Let $f_{l}$ be the density of $P_{l}$ with respect to a suitable reference measure $\lambda$ on $(\mathcal{X}, \mathcal{B})$. Most commonly, $\lambda$ is either the Lebesgue measure (continuously distributed $X_{i}$ ) or the counting measure (discretely distributed $X_{i}$.

Definition 1.1: The stochastic process $(X, Y)$ is a hidden Markov model if there is a (measurable) function $h$ such that for each $n, X_{n}=h\left(Y_{n}, e_{n}\right)$, where $e_{1}, e_{2}, \ldots$ are i.i.d. and independent of $Y$.

Hence, the emission distribution $P_{l}$ is the distribution of $h\left(l, e_{n}\right)$. The distribution of $X$ is completely determined by $\mathbb{P}$ and the emission distributions $P_{l}, l \in S$. It can be shown that $X$ is also ergodic [1], [2], [3]. Let $x_{1: n}=\left(x_{1}, \ldots, x_{n}\right)$ and $y_{1: n}=\left(y_{1}, \ldots, y_{n}\right)$ be fixed observed and unobserved realizations, respectively, of HMM $\left(X_{i}, Y_{i}\right)_{i \geq 1}$ up to time $n$.

J. Lember is with the Institute of Mathematical Statistics, Tartu University, J. Liivi 2-507, 50409, Estonia.

E-mail: jyri@ut.ee

A. Koloydenko is with the Division of Statistics of Nottingham University, University Park, Nottingham, NG7 2RD, UK.

E-mail: alexey.koloydenko@nottingham.ac.uk

Manuscript received April 8, 2008; revised ??, 2008
Treating $y_{1: n}$ as parameters to be estimated, let $\Lambda\left(q_{1: n} ; x_{1: n}\right)$ be the likelihood function $\mathbf{P}\left(Y_{1: n}=q_{1: n}\right) \prod_{i=1}^{n} f_{q_{i}}\left(x_{i} ; \theta_{q_{i}}\right)$ of $q_{1: n}$, and let $\mathcal{V}\left(x_{1: n}\right)$ be the set of the maximum-likelihood estimates $v\left(x_{1: n}\right) \in S^{n}$ of $y_{1: n}$. The elements of $\mathcal{V}\left(x_{1: n}\right)$ are called (Viterbi) alignments and are commonly computed by the Viterbi algorithm [4], [5]. If $\mathbf{P}\left(Y_{1: n}=q_{1: n}\right)$ is thought of as the prior distribution of $Y_{1: n}$, then $v\left(x_{1: n}\right)$ 's also maximize the probability mass function of the posterior distribution of $Y$, hence the term maximum a posteriori $(M A P)$ paths. Besides their direct significance for prediction of $Y$ from $X$, Viterbi alignments, or MAP paths, are also central to the theory and applications of HMMs [6] in the more general setting when any parameters of the emission distributions $P_{l}$ and any of the transition probabilities $p_{i j}, i, j \in S$, would also be unknown and of interest. Therefore, asymptotic behavior of Viterbi alignments is also crucial for the inference on the unknown parameters [6], [7].

To appreciate that the question of extending $v\left(x_{1: n}\right)$ ad infinitum is not a trivial one even if the problem of nonuniqueness of $v\left(x_{1: n}\right)$ is disregarded, suffice it to say that an additional observation $x_{n+1}$ can in principle change the entire alignment based on $x_{1: n}$, i.e. $v\left(x_{1: n}\right)$ and $v\left(x_{1: n+1}\right)_{1: n}$ can disagree significantly, if not fully. Fortunately, the situation is not hopeless and in this paper we prove that in most HMMs alignments can be consistently extended piecewise. Specifically, motifs of (contiguous) observations $z_{1: b}$, called barriers, are observed with positive probability, forcing Viterbi alignments based on extended observations $\left(x_{1: n}, z_{1: b}, x_{n+b+1: n+b+r}\right), n \geq 0, r \geq 1$, to stabilize as follows: Roughly, $v\left(x_{1: n} z_{1: b} x_{n+b+1: n+b+r}\right)_{1: n}=v\left(x_{1: n}\right)$ for all $x_{1: n}$ and all extensions $x_{n+b+1: n+b+r}$. To be more precise, a particular state $l \in S$ and an element $b_{k}$, called a node, of the barrier $b$ can be found such that regardless of the observations before and after $b$, the alignment has to go through $l$ at time $u=n+k$. The optimality principle then insures the stabilization $v\left(x_{1: n} z_{1: b} x_{n+b+1: n+b+r}\right)_{1: u}=v\left(x_{1: u}\right)$ and in particular $v_{u}=l$.

Suppose now that $x_{1: n}$ contains several barriers with nodes occurring at times $u_{1}<\cdots<u_{m} \leq n$. Then the Viterbi alignment $v\left(x_{1: n}\right)$ can be constructed piecewise as follows: Let $v\left(x_{1: \infty}\right)=\left(v^{1}, v^{2}, \ldots, v^{m}, v^{m+1}\right)$, where $v^{1}$ is the alignment based on $x_{1: u_{1}}$ and ending in $l$, and let $v^{i}$, for $i=2,3, \ldots, m+$ 1 , be the conditional alignment based on $x_{u_{i-1}: u_{i}}$ given that $Y_{u_{i-1}}=l$; note that the alignments $v^{i}, i=2,3, \ldots, m$ also end in $l$. Now, if a new observation $x_{n+1}$ is added, then the last segment $v^{m+1}$ can change, but the segments $v^{1}, \ldots, v^{m}$ are intact. Suppose now that a realization $x_{1: \infty}$ contains infinitely many barriers, and hence also infinitely many nodes. Then the 
(piecewise) infinite alignment $v\left(x_{1: \infty}\right)$ is defined naturally as the infinite succession of the segments $v^{1}, v^{2}, \ldots$

In this paper, we prove that for some fixed integer $M>0$, the probability that the finite random process $X_{1: M}$ generates a barrier, is positive. Since $X$ is ergodic, almost every realization $x_{1: \infty}$ has infinitely many barriers and, therefore, the infinite piecewise alignment is well-defined. Apparently, the piecewise alignment gives rise to a decoding process $v: \mathcal{X}^{\infty} \mapsto S^{\infty}$ via $V_{1: \infty}=v\left(X_{1: \infty}\right)$, which we shall call the Viterbi alignment process. The construction ensures that $V$ is regenerative and ergodic. Note also how this piecewise construction naturally calls for a buffered on-line implementation in which the memory used to store $x_{u_{i-1}: u_{i}}$ can be released once $v^{i}$ has been computed.

\section{A. Previous related work and contribution of this work}

The problem of constructing infinite Viterbi processes has been brought to the attention of the IEEE Information Theory community fairly recently by [8] and [9]. Although the piecewise structure of Viterbi alignments was already acknowledged in [10], to our best knowledge, the subject has been first seriously considered in [8], [9]. In these latter works, the existence of infinite alignments for certain special cases, such as $K=2$ and Markov chains with additive white Gaussian noise, has been proved. In particular, in these cases the authors of [8], [9] have proved the existence of 'meeting times' and 'meeting states', which are a special (stronger) type of nodes. While innovative, the main result of [8] (Theorem 2) makes several restrictive assumptions and is proved in an existential manner, which prevents its extension beyond the $K=2$ case.

Independently of these works, [11], [7], [12] have developed a more general theory to include the problem of estimating unknown parameters $\left(\theta_{i}\right.$, and $\left.p_{i j}, i, j \in S\right)$. Namely, the focus of this theory has been the Viterbi training (VT), or extraction, algorithm [13]. Competing with EM-based procedures, this algorithm provides computationally and intuitively appealing estimates which, on the other hand, are biased, even in the limit when $n \rightarrow \infty$. In order to reduce this bias, the adjusted Viterbi training (VA) has been introduced in [11], [7], [12]. Naturally, VA relies on the existence of infinite alignments and their ergodic properties. Although the general theory has been presented in [12], [7], some of the main results of the theory (Lemma 3.1 and 3.2 of [7]) have appeared without proof due to the limitations of scope and size. This paper slightly refines these results and, most importantly, presents their complete proofs. Whereas these results are formulated for general HMMs ( $K \geq 2)$, [14] has most recently considered in full detail the special case of $K=2$, generalizing similar results of [8], [9]. Specifically, it has been proved in [14] that infinitely many barriers (and hence the infinite Viterbi alignment) exist for any aperiodic and irreducible 2-state $H M M$. Thus, the results presented here generalize the ones of [14] and [8], [9] for $K \geq 2$. It turns out that this generalization is far from being straightforward and requires a more advanced analysis and tools. Furthermore, as we show below, when $K>2$, not every aperiodic and irreducible HMM has infinitely many nodes, undermining the piecewise construction of infinite alignments for those models. The disappearance of nodes is due to the fact that an aperiodic and irreducible Markov chain can have zeros in the transition matrix. If this possibility is excluded, as is the case in [8], [9], the 'meeting times' and 'meeting states' of [8], [9] are sufficient to prove the existence of infinite Viterbi alignments for many HMMs used in practice. In their recent communication with us, the authors of [8], [9] have corrected those statements in their above works where the strict positivity of the transition matrix is implicitly assumed but formally omitted (see [7] for details). At the same time, in order to accommodate for zeros in the transition matrix, [7] introduced a more general notion of nodes, effectively removing the limitations of the notion of 'meeting times' and 'meeting states'. However, the price for this generalization has been rather high due to the interfering issue of non-uniqueness of (finite) Viterbi alignments. For a detailed treatment of the piecewise construction of the infinite alignment and process in general HMMs, and the role of the infinite Viterbi process for the adjusted Viterbi training theory, we refer to the state-of-the-art article [7].

\section{B. Organization of the rest of the paper}

In $\S$ II we briefly outline the construction of the infinite alignments $\S$ II-B based on [7]. This includes definitions of nodes $\S$ II-A and barriers $\S$ II-C. Next, $§$ III states our main results which have first appeared in [7] and guarantee the existence of the alignment process $V$. In $\S$ III-B, we give a counterexample to explain the necessity of our technical assumptions. In $\S \mathrm{IV}$, we present a complete and detailed proof of our main results. This is followed in $\S \mathrm{V}$ by a brief discussion of the significance of the presented results.

\section{Construction}

\section{A. Nodes}

First, consider the scores

$$
\delta_{u}(l) \stackrel{\text { def }}{=} \max _{q \in S^{u-1}} \Lambda\left((q, l) ; x_{1: u}\right) .
$$

Thus, $\delta_{u}(l)$ is the maximum of the likelihood of the paths terminating at $u$ in state $l$. Note that $\delta_{1}(l)=\pi_{l} f_{l}\left(x_{1}\right)$ and the recursion below

$$
\delta_{u+1}(j)=\max _{l \in S}\left(\delta_{u}(l) p_{l j}\right) f_{j}\left(x_{u+1}\right) \quad \forall u \geq 1, \forall j \in S,
$$

helps to verify that $\mathcal{V}\left(x_{1: n}\right)$, the set of all the Viterbi alignments, can be written as follows: $\mathcal{V}\left(x_{1: n}\right)=$ $\left\{v \in S^{n}: \forall i \in S, \delta_{n}\left(v_{n}\right) \geq \delta_{n}(i)\right.$ and

$\left.\forall u: 1 \leq u<n, v_{u} \in t\left(u, v_{u+1}\right)\right\}$, where $\forall u \geq 1, \forall j \in S$,

$$
t(u, j) \stackrel{\text { def }}{=}\left\{l \in S: \forall i \in S \delta_{u}(l) p_{l j} \geq \delta_{u}(i) p_{i j}\right\} .
$$

Next, we introduce $p_{i j}^{(r)}(u)$, the maximum of the likelihood realized along the paths connecting states $i$ and $j$ at times $u$ and $u+r$, respectively. Thus, $p_{i j}^{(0)}(u) \stackrel{\text { def }}{=}$ $p_{i j}$ and $\forall u \geq 1$, and $\forall r \geq 1$, let $p_{i j}^{(r)}(u) \stackrel{\text { def }}{=}$ $\max _{q_{1: r} \in S^{r}} p_{i q_{1}} f_{q_{1}}\left(x_{u+1}\right) p_{q_{1} q_{2}} f_{q_{2}}\left(x_{u+2}\right) p_{q_{2} q_{3}} \cdots$

$$
\cdots p_{q_{r-1} q_{r}} f_{q_{r}}\left(x_{u+r}\right) p_{q_{r} j} .
$$


Note also

$$
\begin{aligned}
\delta_{u+1}(j) & =\max _{i \in S}\left\{\delta_{u-r}(i) p_{i j}^{(r)}(u-r)\right\} f_{j}\left(x_{u+1}\right) \quad \forall r<u \\
p_{i j}^{(r)}(u) & =\max _{q \in S} p_{i q}^{(r-1)}(u) f_{q}\left(x_{u+r}\right) p_{q j} .
\end{aligned}
$$

Definition 2.1: Let $0 \leq r<n, u \leq n-r$ and let $l \in S$. Given $x_{1: u+r}$, the first $u+r$ observations, $x_{u}$ is said to be an $l$-node of order $r$ if

$$
\delta_{u}(l) p_{l j}^{(r)}(u) \geq \delta_{u}(i) p_{i j}^{(r)}(u) \quad \forall i, j \in S .
$$

Also, $x_{u}$ is said to be a node of order $r$ if it is an $l$-node of order $r$ for some $l \in S ; x_{u}$ is said to be a strong node of order $r$ if the inequalities in (5) are strict for every $i, j \in S, i \neq l$. Let $x_{1: n}$ be such that $x_{u_{i}}$ is an $l_{i}$-node of order $r, 1 \leq i \leq k$, for some $k<n$, and assume $u_{k}+r<n$ and $u_{i+1}>u_{i}+r$ for all $i=1,2, \ldots, k-1$. Such nodes are said to be separated.

\section{B. Piecewise alignment}

Suppose $x_{1: n}$ is such that for some $u_{i}, r_{i}, i=1,2, \ldots, k$, $u_{1}+r_{1}<u_{2}+r_{2}<\cdots<u_{k}+r_{k}<n, x_{u_{i}}$ is an $l_{i^{-}}$ node of order $r_{i}$. It follows then easily from the definition of the node that there exists a Viterbi alignment $v\left(x_{1: n}\right) \in$ $\mathcal{V}\left(x_{1: n}\right)$ that goes through $l_{i}$ at $u_{i}$ (i.e. $v_{u_{i}}=l_{i}$ ) for each $i=1,2, \ldots, k$ (see [7]). It is not difficult to verify that such $v\left(x_{1: n}\right)$ can actually be computed as follows: Obtain $v^{1}$, a path that is optimal among all those that end at $u_{1}$ in $l_{1}$. (Note that unless the order of the node $x_{u_{1}}$ is $0, v^{1}$ need not be in $\mathcal{V}\left(x_{1: u_{1}}\right)$.) Given $x_{u_{1}+1: u_{2}}$, continue on by taking $v^{2}$ to be a maximum likelihood path from $l_{1}$ to $l_{2}$. That is, $v^{2}$ maximizes the constrained likelihood under the initial distribution $\left(p_{l_{1}}\right.$.) and the constraint $v_{u_{2}-u_{1}}^{2}=l_{2}$. Now, $\left(v^{1}, v^{2}\right)$ maximizes the likelihood given $x_{1: u_{2}}$ over all paths ending with $l_{2}$. Similarly, we define the pieces $v^{3}, \ldots, v^{k}$. Finally, $v^{k+1}$ is chosen to maximize the (unconstrained) likelihood given $x_{u_{k+1}: n}$ under the initial distribution $\left(p_{l_{k}}\right.$.).

The separated nodes assumption $u_{i+1}>u_{i}+r, 1 \leq i<k$, is not restrictive at all since it is always possible to choose from any infinite sequence of nodes an infinite subsequence of separated ones. The reason for this requirement has to do with the non-uniqueness of alignments and is as follows. The fact that $x_{u_{i}}$ is an $r$ th order $l_{i}$-node guarantees that when backtracking from $u_{i}+r$ down to $u_{i}$, ties (if any) can be broken in such a way that, regardless of the values of $x_{u_{i}+r+1: n}$ and how ties are broken in between $n$ and $u_{i}+r$, the alignment goes through $l_{i}$ at $u_{i}$. At the same time, segment $u_{i}, \ldots, u_{i}+r$ is 'delicate', that is, unless $x_{u_{i}}$ is a strong node, breaking the ties arbitrarily within $u_{i}, \ldots, u_{i}+r$ can result in $v_{u_{i}} \neq l_{i}$. Hence, when neither $x_{u_{i}}$ nor $x_{u_{i+1}}$ is strong and $u_{i+1} \leq u_{i}+r$, breaking the ties in favor of $x_{u_{i}}$ can result in $v_{u_{i+1}} \neq l_{i+1}$. Clearly, such a pathological situation is impossible if $r=0$ and might also be rare in practice even for $r>0$.

\footnotetext{
${ }^{1}$ Note that if $x_{u}$ is a node of order $r$, it is then also a node of any order higher than $r$. Hence, the order of a node is defined to be the minimum such
} $r$.
To formalize the piecewise construction, let

$$
\begin{aligned}
& \mathcal{W}^{l}\left(x_{1: n}\right) \stackrel{\text { def }}{=}\left\{v \in S^{n}: v_{n}=l\right. \\
& \left.\Lambda\left(v ; x_{1: n}\right) \geq \Lambda\left(w ; x_{1: n}\right) \quad \forall w \in S^{n}: w_{n}=l\right\}
\end{aligned}
$$

$\mathcal{V}^{l}\left(x_{1: n}\right) \stackrel{\text { def }}{=}\left\{v \in \mathcal{V}\left(x_{1: n}\right): v_{n}=l\right\}$ be the set of maximizers of the constrained likelihood, and the subset of maximizers of the (unconstrained) likelihood, respectively, all elements of which go through $l$ at $n$. Note that unlike $\mathcal{W}^{l}\left(x_{1: n}\right), \mathcal{V}^{l}\left(x_{1: n}\right)$ might be empty. It can be shown that $\mathcal{V}^{l}\left(x_{1: n}\right) \neq \emptyset \Rightarrow \mathcal{V}^{l}\left(x_{1: n}\right)=\mathcal{W}^{l}\left(x_{1: n}\right)$. Also, let subscript the $(l)$ in $\mathcal{W}_{(l)}^{m}\left(x_{1: n}\right)$ and $\mathcal{V}_{(l)}\left(x_{1: n}\right)$ refer to $\left(p_{l i}\right)_{i \in S}$ being used as the initial distribution in place of $\pi$. With these notations, the piecewise alignment is $v=\left(v^{1}, \ldots, v^{k+1}\right) \in \mathcal{V}\left(x_{1: n}\right)$, where

$$
\begin{aligned}
v^{1} & \in \mathcal{W}^{l_{1}}\left(x_{1: u_{1}}\right), \quad v^{k+1} \in \mathcal{V}_{\left(l_{k}\right)}\left(x_{u_{k}+1: u_{n}}\right) \\
v^{i} & \in \mathcal{W}_{\left(l_{i-1}\right)}^{l_{i}}\left(x_{u_{i-1}+1: u_{i}}\right), \quad 2 \leq i \leq k .
\end{aligned}
$$

Moreover, for $i=1,2, \ldots, k$, the partial paths $w(i) \stackrel{\text { def }}{=}$ $\left(v^{1}, \ldots, v^{i}\right) \in \mathcal{W}^{l_{i}}\left(x_{1: u_{i}}\right)$.

If $x_{1: \infty}$ has infinitely many (separated) nodes $\left\{x_{u_{k}}\right\}_{k \geq 1}$ then $v\left(x_{1: \infty}\right)$, an infinite piecewise alignment based on the node times $\left\{u_{k}\left(x_{1: \infty}\right)\right\}_{k \geq 1}$ can be defined as follows: If the sets $\mathcal{W}_{\left(l_{i-1}\right)}^{l_{i}}\left(x_{u_{i-1}+1: u_{i}}\right), i=2, \ldots, k$ as well as $\mathcal{V}_{\left(l_{k}\right)}\left(x_{u_{k}+1: n}\right)$ and $\mathcal{W}^{l_{1}}\left(u_{1}, x_{1: u_{1}}\right)$ are singletons, then (6) immediately defines a unique infinite alignment $v\left(x_{1: \infty}\right)=$ $\left(v^{1}\left(x_{1: u_{1}}\right), v^{2}\left(x_{u_{1}+1: u_{2}}\right), \ldots\right)$. Otherwise, ties must be broken. If we want our infinite alignment process $V$ to be regenerative (see [7]), a natural consistency condition must be imposed on rules to select unique $v\left(x_{1: n}\right)$ from $\mathcal{W}^{l_{1}}\left(x_{1: u_{1}}\right) \times$ $\mathcal{W}_{\left(l_{1}\right)}^{l_{2}}\left(x_{u_{1}+1: u_{2}}\right) \times \cdots \times \mathcal{W}_{\left(l_{k-1}\right)}^{l_{k}}\left(x_{u_{k-1}+1: u_{k}}\right) \times \mathcal{V}_{\left(l_{k}\right)}\left(x_{u_{k}+1: n}\right)$. In [7], resulting infinite alignments, as well as decoding $v: \mathcal{X}^{\infty} \rightarrow S^{\infty}$ based on such alignments, are called proper. This condition is, perhaps, best understood by the following example. Suppose for some $x_{1: 5} \in \mathcal{X}^{5}, \mathcal{W}_{(1)}^{1}\left(x_{1: 5}\right)=$ $\{12211,11211\}$, and suppose the tie is broken in favor of 11211. Now, whenever $\mathcal{W}_{(l)}^{1}\left(x_{1: 4}^{\prime}\right)$ contains $\{1221,1121\}$, we naturally require that 1221 not be selected. In particular, we select 1121 from $\mathcal{W}_{(1)}^{1}\left(x_{1: 4}\right)=\{1221,1121\}$. Subsequently, 112 is selected from $\mathcal{W}_{(1)}^{2}\left(x_{1: 3}\right)=\{122,112\}$, and so on. It can be shown that a decoding by piecewise alignment (6) with ties broken in favor of min (or max) under the reverse lexicographic ordering of $S^{n}, n \in \mathbb{N}$, is a proper decoding.

Note also that we break ties locally, i.e. within individual intervals $u_{i-1}+1, \ldots, u_{i}, i \geq 2$, enclosed by adjacent nodes. This is in contrast to global ordering of $\mathcal{V}\left(x_{1: n}\right)$, such as the one in [8], [9]. Since a global order need not respect decomposition (6), it can fail to produce an infinite alignment going through infinitely many nodes unless the nodes are strong.

\section{Barriers}

Recall (Definition 2.1) that nodes of order $r$ at time $u$ are defined relative to the entire realization $x_{1: u+r}$. Thus, whether $x_{u}$ is a node or not depends, in principle, on all observations up to $x_{u}$.

We show below that typically a block $x_{1: k}^{b} \in \mathcal{X}^{k}(k \geq r)$ can be found such that for any $w \geq 1$ and for any $x_{1: w}^{\prime} \in \mathcal{X}^{w}$, 
$(w+k-r)$ th element of $\left(x_{1: w}^{\prime}, x_{1: k}^{b}\right)$ is a node of order $r$ (relative to $\left.\left(x_{1: w}^{\prime}, x_{1: k}^{b}\right)\right)$. Sequences $x_{1: k}^{b}$ that ensure existence of such persistent nodes are called barriers in [7]. Specifically,

Definition 2.2: Given $l \in S, x_{1: k}^{b} \in \mathcal{X}^{k}$ is called an (strong) l-barrier of order $r \geq 0$ and length $k \geq 1$ if, for any $w \geq 1$ and for every $x_{1: w}^{\prime} \in \mathcal{X}^{w},\left(x_{1: w}^{\prime}, x_{1: k}^{b}\right)$ is such that $\left(x_{1: w}^{\prime}, x_{1: k}^{b}\right)_{w+k-r}$ is an (strong) $l$-node of order $r$.

\section{EXISTENCE}

\section{A. Clusters and main results}

For each $i \in S$, let

$$
G_{i} \stackrel{\text { def }}{=}\left\{x \in \mathcal{X}: f_{i}(x)>0\right\} .
$$

Definition 3.1: We call a subset $C \subset S$ a cluster if the following conditions are satisfied:

$$
\min _{j \in C} P_{j}\left(\cap_{i \in C} G_{i}\right)>0, \text { and } \max _{j \notin C} P_{j}\left(\cap_{i \in C} G_{i}\right)=0 .
$$

Hence, a cluster is a maximal subset of states such that $G_{C}=$ $\cap_{i \in C} G_{i}$, the intersection of the supports of the corresponding emission distributions, is 'detectable'. Distinct clusters need not be disjoint and a cluster can consist of a single state. In this latter case such a state is not hidden, since it is exposed by any observation it emits. When $K=2, S$ is the only cluster possible, since otherwise all observations would expose their states and the underlying Markov chain would cease to be hidden. In practice, many other HMMs have the entirety of $S$ as their (necessarily unique) cluster.

We now state the main results. For every state $l \in S$, let

$$
p_{l}^{*}=\max _{j} p_{j l} .
$$

Lemma 3.1: Assume that for each state $l \in S$,

$$
P_{l}\left(\left\{x \in \mathcal{X}: f_{l}(x) p_{l}^{*}>\max _{i, i \neq l} f_{i}(x) p_{i}^{*}\right\}\right)>0 .
$$

Moreover, assume that there exists a cluster $C \subset S$ and a positive integer $m$ such that the $m$ th power of the substochastic matrix $\mathbb{Q}=\left(p_{i j}\right)_{i, j \in C}$ is strictly positive. Then, for some integers $M$ and $r, M>r \geq 0$, there exist a set $B=B_{1} \times \cdots \times B_{M} \subset \mathcal{X}^{M}$, an $M$-tuple of states $q_{1: M} \in S^{M}$ and a state $l \in S$, such that every $x_{1: M} \in B$ is an $l$-barrier of order $r$ (and length $M$ ), $q_{M-r}=l$ and

$$
\mathbf{P}\left(X_{1: M} \in B, \quad Y_{1: M}=q_{1: M}\right)>0 .
$$

Lemma 3.1 implies that $\mathbf{P}\left(X_{1: M} \in B\right)>0$. Also, since every element of $B$ is a barrier of order $r$, the ergodicity of $X$ therefore guarantees that almost every realization of $X$ contains infinitely many l-barriers of order $r$. Hence, almost every realization of $X$ also has infinitely many l-nodes of order $r$.

In two state HMMs, $S$ is the only cluster (otherwise the Markov chain would not be hidden), hence $\mathbb{Q}=\mathbb{P}$. The irreducibility and aperiodicity in this case imply strict positivity of $\mathbb{P}^{2}$. Thus, the only condition to be verified is (8), which in this case writes as $P_{1}\left(\left\{x \in \mathcal{X}: f_{1}(x) p_{1}^{*}>f_{2}(x) p_{2}^{*}\right\}\right)>0$ and
$P_{2}\left(\left\{x \in \mathcal{X}: f_{2}(x) p_{2}^{*}>f_{1}(x) p_{1}^{*}\right\}\right)>0$. In [14], it is shown that in the case of two state HMMs, one of these two positivity conditions is always met, which, in fact, turns out to be sufficient for the existence of infinitely many strong barriers in this $(K=2)$ case. Thus, any two state HMM with irreducible and aperiodic $Y$ has infinitely many strong barriers. Lemma 3.1 significantly generalizes this and associated results of [14]. The case $K=2$ is special in several respects, hence the generalization is technically involved, and in particular the CLT-based proof of the existence of infinitely many nodes in [8] (Theorem 2) does not apply when $K>2$.

For certain technical reasons, instead of extracting subsequences of separated nodes from general infinite sequences of nodes guaranteed by Lemma 3.1, we achieve node separation by adjusting the notion of barriers. Namely, note that two $r$ thorder $l$-barriers $x_{j: j+M-1}$ and $x_{i: i+M-1}$ might be in $B$ with $j<i \leq j+r$, implying that the associated nodes $x_{j+M-r-1}$ and $x_{i+M-r-1}$ are not separated. Thus, we impose on $B$ the following condition:

$$
x_{j: j+M-1}, x_{i: i+M-1} \in B, i \neq j \Rightarrow|i-j|>r .
$$

If (9) holds, we say that the barriers from $B \subset \mathcal{X}^{M}$ are separated. This is often easy to achieve by a simple extension of $B$ as shown in the following example. Suppose there exists $x \in \mathcal{X}$ such that $x \notin B_{m}$, for all $m=1,2, \ldots, M$. All elements of $B^{*} \stackrel{\text { def }}{=}\{x\} \times B$ are evidently barriers, and moreover, they are now separated. The following Lemma incorporates a more general version of the above example.

Lemma 3.2: Suppose the assumptions of Lemma 3.1 are satisfied. Then, for some integers $M$ and $r, M>r \geq 0$, there exist $B=B_{1} \times \cdots \times B_{M} \subset \mathcal{X}^{M}, q_{1: M} \in S^{M}$, and $l \in S$, such that every $x_{1: M}^{b} \in B$ is a separated $l$-barrier of order $r$ (and length $M), q_{M-r}=l$, and $\mathbf{P}\left(X_{1: M} \in B, \quad Y_{1: M}=q_{1: M}\right)>$ 0 .

\section{B. Counterexamples}

The condition on $C$ in Lemma 3.1 might seem technical and even unnecessary. We next give an example of an HMM where the cluster condition is not met and no node (barrier) can occur. Then, we will modify the example to enforce the cluster condition and consequently gain barriers.

Example 3.2: Let $K=4$ and consider an ergodic Markov chain with transition matrix

$$
\mathbb{P}=\left(\begin{array}{cccc}
\frac{1}{2} & 0 & 0 & \frac{1}{2} \\
0 & \frac{1}{2} & \frac{1}{2} & 0 \\
\frac{1}{2} & 0 & \frac{1}{2} & 0 \\
0 & \frac{1}{2} & 0 & \frac{1}{2}
\end{array}\right)
$$

Let the emission distributions be such that (8) is satisfied and $G_{1}=G_{2}$ and $G_{3}=G_{4}$ and $G_{1} \cap G_{3}=\emptyset$. Hence, in this case there are two disjoint clusters $C_{1}=\{1,2\}, C_{2}=\{3,4\}$. The matrices $\mathbb{Q}_{i}$ corresponding to $C_{i}, i=1,2$ are

$$
\mathbb{Q}_{1}=\mathbb{Q}_{2}=\left(\begin{array}{cc}
\frac{1}{2} & 0 \\
0 & \frac{1}{2}
\end{array}\right) .
$$

Evidently, the cluster assumption of Lemma 3.1 is not satisfied. Note also that the alignment cannot change (in one step) 
its state to the opposite one within the same cluster. Since the supports $G_{1,2}$ and $G_{3,4}$ are disjoint, any observation exposes the corresponding cluster. Hence any sequence of observations can be regarded as a sequence of blocks emitted from alternating clusters. However, the alignment inside each block stays constant. It can be shown that in this case no $x_{u}$ can be a node (of any order) for any $n>1, x_{1: n} \in \mathcal{X}^{n}$, and $1 \leq u<n$.

Let us modify the HMM in Example 3.2 to ensure the assumptions of Lemma 3.1.

Example 3.3: Let $\epsilon$ be such that $0<\epsilon<\frac{1}{2}$ and let us replace $\mathbb{P}$ by the following transition matrix

$$
\left(\begin{array}{cccc}
\frac{1}{2}-\epsilon & \epsilon & 0 & \frac{1}{2} \\
\epsilon & \frac{1}{2}-\epsilon & \frac{1}{2} & 0 \\
\frac{1}{2} & 0 & \frac{1}{2} & 0 \\
0 & \frac{1}{2} & 0 & \frac{1}{2}
\end{array}\right)
$$

Let the emission distributions be as in the previous example. In this case, the cluster $C_{1}$ satisfies the assumption of Lemma 3.1. As previously, every observation exposes its cluster. Lemma 3.1 now applies to guarantee barriers and nodes. To be more specific, let $\epsilon=1 / 4, f_{1}(x)=\exp (-x)_{x \geq 0}$, $f_{2}(x)=2 \exp (-2 x)_{x \geq 0}$, and $f_{3}(x)=\exp (x)_{x \leq 0}, f_{4}(x)=$ $2 \exp (2 x)_{x \leq 0}$. It can then be verified that if $x_{1: 2}=(1,1)$ then $x_{1}$ is a 1 -node of order 2 . Indeed, in that case any element of $B=(0,+\infty) \times(\log (2),+\infty) \times(0,+\infty)$ is a 1-barrier of order 2 .

Another way to modify the HMM in Example 3.2 to enforce the assumptions of Lemma 3.1 is to change the emission probabilities. Namely, assume that the supports $G_{i}$, $i=1, \ldots, 4$ are such that $P_{j}\left(\cap_{i=1}^{4} G_{i}\right)>0$ for all $j \in S$, and (8) holds. Now, $S=\{1, \ldots, 4\}$ is the only cluster. Since the matrix $\mathbb{P}^{2}$ has all its entries positive, the conditions of Lemma 3.1 are now satisfied and barriers can now be constructed.

\section{PROOF OF THE MAIN RESULT}

\section{A. Proof of Lemma 3.1}

The proof below is a rather direct construction which is, however, technically involved. In order to facilitate the exposition of this proof, we have divided it into 17 short parts as follows.

1) $\mathcal{X}_{l} \subset \mathcal{X}:$ It follows from the assumption (8) and finiteness of $S$ that there exists an $\epsilon>0$ such that for all $l \in S P_{l}\left(\mathcal{X}_{l}\right)>0$, where

$$
\mathcal{X}_{l} \stackrel{\text { def }}{=}\left\{x \in \mathcal{X}: \max _{i, i \neq l} p_{i}^{*} f_{i}(x)<(1-\epsilon) p_{l}^{*} f_{l}(x)\right\} .
$$

(Note that $p_{l}^{*}>0$ for all $l \in S$ by irreducibility of $Y$.) Also note that $\mathcal{X}_{l}, l \in S$ are disjoint and have positive reference measure $\lambda\left(\mathcal{X}_{l}\right)>0$.

2) $\mathcal{Z} \subset \mathcal{X}$ and $\delta-K$ bounds on cluster densities $f_{i}, i \in C$ : Let $C$ be a cluster as in the assumptions of the Lemma. The existence of $C$ implies the existence of a set $\hat{\mathcal{Z}} \subset \cap_{i \in C} G_{i}$ and $\delta>0$, such that $\lambda(\hat{\mathcal{Z}})>0$, and $\forall z \in \hat{\mathcal{Z}}$, the following statements hold:

(i) $\min _{i \in C} f_{i}(z)>\delta$;

(ii) $\max _{j \notin C} f_{j}(z)=0$.
Indeed, $\min _{j \in C} P_{j}\left(\cap_{i \in C} G_{i}\right)>0$ implies (and indeed is equivalent to) $\lambda\left(\cap_{i \in C} G_{i}\right)>0$. The latter implies the existence of $\hat{\mathcal{Z}} \subset \cap_{i \in C} G_{i}$ with positive $\lambda$-measure and $\delta>0$ such that (i) holds. Since $\lambda\left(\cap_{i \in C} G_{i}\right)>0$, the condition $P_{j}\left(\cap_{i \in C} G_{i}\right)=0$ for $j \notin C$ implies (is equivalent to) $f_{j}=0$ $\lambda$-almost everywhere on $\cap_{i \in C} G_{i}$. Thus, $\max _{j \notin C} f_{j}=0 \lambda$ almost everywhere on $\cap_{i \in C} G_{i}$, which implies (ii).

Evidently, $K>0$ can be chosen sufficiently large to make $\lambda\left(\left\{z \in \mathcal{X}: f_{i}(z) \geq K\right\}\right)$ arbitrarily small, and in particular, to guarantee that $\lambda\left(\left\{z \in \mathcal{X}: f_{i}(z) \geq K\right\}\right)<\frac{\lambda(\hat{\mathcal{Z}})}{|C|}$, where $|C|$ is the size of $C$. Clearly then, redefining $\hat{\mathcal{Z}} \stackrel{\text { def }}{=} \hat{\mathcal{Z}} \cap\{z \in$ $\left.\mathcal{X}: f_{i}(z)<K, i \in C\right\}$ preserves $\lambda(\hat{\mathcal{Z}})>0$. Next, consider

$$
\lambda\left(\hat{\mathcal{Z}} \backslash\left(\cup_{l \in S} \mathcal{X}_{l}\right)\right) \text {. }
$$

If (11) is positive, then define

$$
\mathcal{Z} \stackrel{\text { def }}{=} \hat{\mathcal{Z}} \backslash\left(\cup_{l \in S} \mathcal{X}_{l}\right) .
$$

If (11) is zero, then there must be $s \in C$ such that

$$
\lambda\left(\hat{\mathcal{Z}} \cap \mathcal{X}_{s}\right)>0
$$

and in this case, let

$$
\mathcal{Z} \stackrel{\text { def }}{=} \hat{\mathcal{Z}} \cap \mathcal{X}_{s} .
$$

Such $s \in S$ must clearly exist since $\lambda(\hat{\mathcal{Z}})>0$ but $\lambda\left(\hat{\mathcal{Z}} \backslash\left(\cup_{l \in S} \mathcal{X}_{l}\right)\right)=0$. To see that $s$ must necessarily be in the cluster $C$, note $\forall s \notin C, f_{s}(z)=0 \forall z \in \hat{\mathcal{Z}}$, which implies $\hat{\mathcal{Z}} \cap \mathcal{X}_{s}=\emptyset$.

3) Sequences $\mathbf{s}, \mathbf{a}$, and $\mathbf{b}$ of states in $S$ : Let us define an auxiliary sequence of states $q_{1}, q_{2}$, and so on, as follows: If (11) is zero, that is, if $\mathcal{Z}=\hat{\mathcal{Z}} \cap \mathcal{X}_{s}$ for some $s \in C$, then define $q_{1}=s$, otherwise let $q_{1}$ be an arbitrary state in $C$. Let $q_{2}$ be a state with maximal probability of transition to $q_{1}$, i.e.: $p_{q_{2} q_{1}}=p_{q_{1}}^{*}$ Suppose $q_{2} \neq q_{1}$. Then find $q_{3}$ with $p_{q_{3} q_{2}}=p_{q_{2}}^{*}$. If $q_{3} \notin\left\{q_{1}, q_{2}\right\}$, find $q_{4}: p_{q_{4} q_{3}}=p_{q_{3}}^{*}$, and so on. Let $U$ be the first index such that $q_{U} \in\left\{q_{1}, \ldots, q_{U-1}\right\}$, that is, $q_{U}=q_{T}$ for some $T<U$. This means that there exists a sequence of states $\left\{q_{T}, \ldots, q_{U}\right\}$ such that

- $q_{T}=q_{U}$

- $q_{T+i}=\arg \max _{j} p_{j q_{T+i-1}}, \quad i=1, \ldots, U-T$.

To simplify the notation and without loss of generality, assume $q_{U}=1$. Reorder and rename the states as follows:

$$
\begin{aligned}
& s_{1} \stackrel{\text { def }}{=} q_{U-1}, s_{2} \stackrel{\text { def }}{=} q_{U-2}, \ldots, s_{i} \stackrel{\text { def }}{=} q_{U-i}, \ldots, \\
& s_{L} \stackrel{\text { def }}{=} q_{T}=1 \quad i=1, \ldots, L \stackrel{\text { def }}{=} U-T, \\
& a_{1} \stackrel{\text { def }}{=} q_{T-1}, a_{2} \stackrel{\text { def }}{=} q_{T-2}, \ldots, a_{P} \stackrel{\text { def }}{=} q_{1},
\end{aligned}
$$

where $P \stackrel{\text { def }}{=} T-1$. Hence,

$$
\begin{aligned}
& \left\{q_{1}, \ldots, q_{T-1}, q_{T}, q_{T+1}, \ldots, q_{U-1}, q_{U}\right\}= \\
& \left\{a_{P}, \ldots, a_{1}, 1, s_{L-1}, \ldots, s_{1}, 1\right\} .
\end{aligned}
$$

Note that if $T=1$, then $P=0$ and $\left\{q_{1}, \ldots, \ldots, q_{U-1}, q_{U}\right\}=$ $\left\{1, s_{L-1}, \ldots, s_{1}, 1\right\}$. We have thus introduced special sequences $\mathbf{a}=\left(a_{1}, a_{2}, \ldots, a_{P}\right)$ and $\mathbf{s}=\left(s_{1}, s_{2}, \ldots, s_{L-1}, 1\right)$. Clearly,

$$
\begin{aligned}
& p_{s_{i-1} s_{i}}=p_{s_{i}}^{*}, i=2, \ldots, L, p_{s_{1}}^{*}=p_{1 s_{1}} \\
& p_{a_{i-1} a_{i}}=p_{a_{i}}^{*}, i=2, \ldots, P, p_{a_{1}}^{*}=s_{L}=1 .
\end{aligned}
$$


Next, we are going to exhibit $\mathbf{b}=\left(b_{1}, \ldots, b_{R}\right)$, another auxiliary sequence for some $R \geq 1$, characterized as follows:

(i) $b_{R}=1$;

(ii) $\exists b_{0} \in C$ such that $p_{b_{0} b_{1}} p_{b_{1} b_{2}} \cdots p_{b_{R-1} b_{R}}>0$;

(iii) if $R>1$, then $b_{i-1} \neq b_{i}$ for every $i=1, \ldots, R$.

Thus, the path $b_{1: R}$ connects cluster $C$ to state 1 in $R$ steps. Let us also require that $R$ be minimum such. Clearly such $\mathbf{b}$ and $b_{0}$ do exist due to irreducibility of $Y$. Note also that minimality of $R$ guarantees (iii) (in the special case of $R=1$ it may happen that $b_{1}=1 \in S$ and $p_{11}>0$, in which case $b_{0}$ can be taken to be also 1 ).

4) Determining $k$ : Let $\mathbb{Q}^{m}$ be the $m$ th power of the substochastic matrix $\mathbb{Q}=\left(p_{i j}\right)_{i, j \in C}$; let $q_{i j}$ be the entries of $\mathbb{Q}^{m}$. By the hypothesis of the Lemma, $q_{i j}>0 \forall i, j \in C$. This means that for every $i, j \in C$, there exists a positive probability path from $i$ to $j$ of length $m$. Let $q_{i j}^{*}$ be the probability of a maximum probability path from $i$ to $j$. In other words, for every $i, j \in C$, there exist states $w_{1}, \ldots, w_{m-1} \in C$ such that

$$
p_{i w_{1}} p_{w_{1} w_{2}} \cdots p_{w_{m-1} w_{m-1}} p_{w_{m-1} j}=q_{i j}^{*}>0 .
$$

Let us define

$$
\begin{aligned}
q & =\min _{i, j \in C} q_{i j}^{*}>0, \quad \text { and } \\
A & =\max _{i \in S} \max _{j \in S}\left\{\frac{p_{i}^{*}}{p_{j i}}: p_{j i}>0\right\},
\end{aligned}
$$

where $p_{i}^{*}$ 's are as defined in (7). Choose $k$ sufficiently large for the following to hold:

$$
(1-\epsilon)^{k-1}<q^{2}\left(\frac{\delta}{K}\right)^{2 m} A^{-R}
$$

where $\epsilon$ is as in (10) and $\delta$ and $K$ are as introduced in $\S I V-A 2$.

5) The s-path: We now fix the state sequence

$$
b_{0}, b_{1}, \ldots, b_{R}, s_{1}, s_{2}, \ldots, s_{2 L k}, a_{1}, \ldots, a_{P},
$$

where $s_{L j+i}=s_{i}, j=1, \ldots, 2 k-1, i=1, \ldots, L$, (and in particular $s_{L j}=1, j=1, \ldots, 2 k$ ). The sequence (19) will be called the s-path. The s-path is a concatenation of $2 k \mathrm{~s}$ cycles $s_{1: L}$, the beginning and the end of which are connected to the cluster $C$ via positive probability paths $\mathbf{b}$ and a, respectively (recall that $a_{P}=q_{1} \in C$ and $b_{R}=1$ by construction). Additionally, the $b_{R}, s_{1}, s_{2}, \ldots, s_{2 L k}, a_{1}, \ldots, a_{P}-$ segment of the $s$-path (19) has the important property (14), i.e. every consecutive transition along this segment occurs with the maximal transition probability given its destination state. (However, $\mathbf{b}$, the beginning of the $s$-path, need not satisfy this property.) The $s$-path is almost ready to serve as $q_{1: M}$ promised by the Lemma and its conversion to $q_{1: M}$ will be completed in $§ I V-A 17$. In fact, the idea of the Lemma and its proof is to exhibit (a cylinder subset of) observations such that once emitted along the $s$-path, these observations would trap the Viterbi backtracking so that the latter winds up on the $s$-path. That will guarantee that an observation corresponding to the beginning of the $s$-path, is a node.
6) The barrier: Consider the following sequence of observations

$$
\begin{aligned}
& z_{0}, z_{1}, \ldots, z_{m}, y_{1}^{\prime}, \ldots, y_{R-1}^{\prime}, y_{0}, y_{1}, \ldots, y_{2 L k}, \\
& y_{1}^{\prime \prime}, \ldots, y_{P}^{\prime \prime}, z_{1}^{\prime}, \ldots, z_{m}^{\prime},
\end{aligned}
$$

where

$$
\begin{aligned}
& z_{0}, z_{i}, z_{i}^{\prime} \in \mathcal{Z}, \quad i=1, \ldots, m ; \\
& y_{i}^{\prime} \in \mathcal{X}_{b_{i}}, \quad i=1, \ldots, R-1 ; \\
& y_{0} \in \mathcal{X}_{1}, \quad y_{i+L j} \in \mathcal{X}_{s_{i}}, \quad j=1, \ldots, 2 k-1, i=1, \ldots, L \\
& y_{i}^{\prime \prime} \in \mathcal{X}_{a_{i}}, \quad i=1, \ldots, P .
\end{aligned}
$$

From this point on throughout $\S \mathrm{IV}-\mathrm{A} 15$, we shall be proving that $y_{L k}$ is a 1 -node of order $(k L+m+P)$, and, therefore, that (20) is a 1-barrier of order $(k L+m+P)$.

First, let $u \geq 2 L k+2 m+1+P+R$ and let $x_{1: u}$ be any sequence of observations containing the sequence (20) in the tail.

7) $\alpha, \beta, \gamma, \eta$ : Recall the definition of the scores $\delta_{u}(i)(1)$ and the maximum partial likelihoods $p_{i j}^{(r)}(u)$ (3). Now, we need to introduce the following abbreviated notation. For any $i, j \in S$ and appropriate $r \geq 0$, let

$$
\begin{aligned}
\delta_{i}\left(y_{l}\right) & \stackrel{\text { def }}{=} \delta_{u-P-m-2 k L+l}(i) \quad \forall l: 0 \leq l \leq 2 k L \\
p_{i j}^{(r)}\left(y_{l}\right) & \stackrel{\text { def }}{=} p_{i j}^{(r)}(u-P-m-2 k L+l), \\
p_{i j}^{(r)}\left(y_{l}^{\prime}\right) & \stackrel{\text { def }}{=} p_{i j}^{(r)}(u-P-m-2 k L-R+l) \quad \forall l: \\
1 & \leq l \leq R-1, \\
\delta_{i}\left(z_{l}\right) & \stackrel{\text { def }}{=} \delta_{u-2 L k-2 m-P-R+l}(i) \quad \forall l: 0 \leq l \leq m, \\
p_{i j}^{(r)}\left(z_{l}\right) & \stackrel{\text { def }}{=} p_{i j}^{(r)}(u-2 L k-2 m-P-R+l), \\
\delta_{i}\left(z_{l}^{\prime}\right) & \stackrel{\text { def }}{=} \delta_{u-m+l}(i) \quad \forall l: 1 \leq l \leq m, \\
p_{i j}^{(r)}\left(z_{l}^{\prime}\right) & \stackrel{\text { def }}{=} p_{i j}^{(r)}(u-m+l) .
\end{aligned}
$$

Also, we will be frequently using the scores corresponding to $z_{0}, y_{1}^{\prime}, y_{L k}$, and $y_{2 L k}$, hence the following further abbreviations:

$$
\alpha_{i} \stackrel{\text { def }}{=} \delta_{i}\left(z_{0}\right), \beta_{i} \stackrel{\text { def }}{=} \delta_{i}\left(z_{m}\right), \gamma_{i} \stackrel{\text { def }}{=} \delta_{i}\left(y_{0}\right), \eta_{i} \stackrel{\text { def }}{=} \delta_{i}\left(y_{L k}\right) .
$$

Note that $\forall j \notin C, f\left(z_{0}\right)=f_{j}\left(z_{l}^{\prime}\right)=f_{j}\left(z_{l}\right)=0, l=1, \ldots, m$ by construction of $\mathcal{Z}$ ( $\left(\mathrm{IV}-\mathrm{A} 2\right.$ ). Hence, $\alpha_{j}=\beta_{j}=0 \forall j \notin C$, and a more general implication is that for every $j \in S$

$$
\begin{aligned}
\beta_{j} & =\max _{i \in C} \alpha_{i} p_{i j}^{(m-1)}\left(z_{0}\right) f_{j}\left(z_{m}\right) \\
& =\alpha_{i_{\beta}(j)} p_{i_{\beta}(j) j}^{(m-1)}\left(z_{0}\right) f_{j}\left(z_{m}\right) \text { for some } i_{\beta}(j) \in C ; \\
\gamma_{j} & =\max _{i \in C} \beta_{i} p_{i j}^{(R-1)}\left(z_{m}\right) f_{j}\left(y_{0}\right) \\
& =\beta_{i_{\gamma}(j)} p_{i_{\gamma}(j) j}^{(R-1)}\left(z_{m}\right) f_{j}\left(y_{0}\right) \text { for some } i_{\gamma}(j) \in C .
\end{aligned}
$$

Also, we will use the following representation of $\eta_{j}$ in terms of $\gamma$ :

$$
\begin{aligned}
\eta_{j} & =\max _{i \in S} \gamma_{i} p_{i j}^{(k L-1)}\left(y_{0}\right) f_{j}\left(y_{k L}\right) \\
& =\gamma_{i_{\eta}(j)} p_{i_{\eta}(j) j}^{(k L-1)}\left(y_{0}\right) f_{j}\left(y_{k L}\right) \text { for some } i_{\eta}(j) \in S .
\end{aligned}
$$


8) Bounds on $\beta$ : Recall ( $(\mathrm{IV}-\mathrm{A} 3)$ that $b_{0} \in C$. We show that for every $j \in S$

$$
\beta_{j}<q^{-1}\left(\frac{K}{\delta}\right)^{m} \beta_{b_{0}} .
$$

Fix $j \in S$ and consider $\alpha_{i_{\beta}(j)}$ from (23). Let $v_{1}, \ldots, v_{m-1}$ be a path that realizes $p_{i j}^{(m-1)}\left(z_{0}\right)$.

Then $\beta_{j}=\alpha_{i_{\beta}(j)} p_{i_{\beta}(j) v_{1}} f_{v_{1}}\left(z_{1}\right) p_{v_{1} v_{2}} f_{v_{2}}\left(z_{2}\right) \cdots$ $p_{v_{m-1} j} f_{j}\left(z_{m}\right)<\alpha_{i_{\beta}(j)} K^{m}$. (The last inequality follows from (12), (13).) Let $w_{1}, \ldots, w_{m-1}$ be a maximum probability path from $i_{\beta(j)}$ to $b_{0}$ as in (15). Thus,

$$
\begin{aligned}
\beta_{b_{0}} \geq \geq & \alpha_{i_{\beta}(j)} p_{i_{\beta}(j) b_{0}}^{(m-1)}\left(z_{0}\right) f_{b_{0}}\left(z_{m}\right) \\
\geq & \alpha_{i_{\beta}(j)} p_{i_{\beta}(j) w_{1}} f_{w_{1}}\left(z_{1}\right) p_{w_{1} w_{2}} f_{w_{2}}\left(z_{2}\right) \cdots \\
& \cdots p_{w_{m-1} b_{0}} f_{b_{0}}\left(z_{m}\right) \geq \alpha_{i_{\beta}(j)} q \delta^{m} .
\end{aligned}
$$

(The last inequality again follows from (12), (13).) Since $q>0$ (16), we thus obtain:

$$
\beta_{j}<\alpha_{i_{\beta}(j)} K^{m} \leq \frac{\beta_{b_{0}}}{q \delta^{m}} K^{m}
$$

as required.

9) Likelihood ratio bounds: We next prove the following claims

$$
\begin{gathered}
p_{i 1}^{(L-1)}\left(y_{l L}\right) \leq p_{11}^{(L-1)}\left(y_{l L}\right) \\
\forall i \in S \quad \forall l=0, \ldots, 2 k-1, \\
\frac{p_{i j}^{(L-1)}\left(y_{l L}\right) f_{j}\left(y_{(l+1) L}\right)}{p_{11}^{(L-1)}\left(y_{l L}\right) f_{1}\left(y_{(l+1) L}\right)}<1-\epsilon \\
\forall i, j \in S, j \neq 1, \forall l: 0 \leq l \leq 2 k-1, \\
p_{i j}^{(R-1)}\left(z_{m}\right) f_{j}\left(y_{0}\right) \leq A^{R} p_{b_{0} 1}^{(R-1)}\left(z_{m}\right) f_{1}\left(y_{0}\right) \\
\forall i, j \in S, \\
\frac{p_{i j}^{(m+P-1)}\left(y_{2 k L}\right)}{p_{1 j}^{(m+P-1)}\left(y_{2 k L}\right)} \leq q^{-1}\left(\frac{K}{\delta}\right)^{m-1} \\
\forall j \in C \forall i \in S .
\end{gathered}
$$

If $L=1$, then (27) becomes $p_{i 1} \leq p_{11}$ for all $i \in S$, which is true by the assumption $p_{1}^{*}=p_{11}$ made in the course of constructing the $\mathrm{s}$ sequence ( $(\mathrm{IV}-\mathrm{A} 3)$. If $L=1$, then (28) becomes

$$
\frac{p_{i j} f_{j}\left(y_{l+1}\right)}{p_{11} f_{1}\left(y_{l+1}\right)}<1-\epsilon \quad \forall i, j \in S, j \neq 1,
$$

and thus, since $y_{l+1} \in \mathcal{X}_{1}, 0 \leq l<2 k$ in this case, (28) is true by the definition of $\mathcal{X}_{1}(\S \mathrm{IV}-\mathrm{A} 1)$ (and the fact that $p_{1}^{*}=p_{11}$ ). Let us next prove (27) and (28) for the case $L>1$. Consider any $l=0,1, \ldots, 2 k-1$. Note that the definitions of the $s$ path (19), $\mathcal{X}_{s_{i}}(\S \mathrm{IV}-\mathrm{A} 1)$, and the fact that $y_{l L+i} \in \mathcal{X}_{s_{i}}$ for $1 \leq i<L$ imply that given observations $y_{L l+1: L(l+1)-1}$, the path $s_{1: L-1}$ realizes the maximum in $p_{11}^{(L-1)}\left(y_{L l}\right)$, i.e.

$$
\begin{aligned}
p_{11}^{(L-1)}\left(y_{l L}\right)= & p_{1 s_{1}} f_{s_{1}}\left(y_{l L+1}\right) p_{s_{1} s_{2}} \ldots \\
& \cdots p_{s_{L-2} s_{L-1}} f_{s_{L-1}}\left(y_{(l+1) L-1}\right) p_{s_{L-1} 1} .
\end{aligned}
$$

(Indeed, $p_{1 s_{1}} f_{s_{1}}\left(y_{l L+1}\right) p_{s_{1} s_{2}} \cdots$

$$
\begin{array}{r}
\cdots p_{s_{L-2} s_{L-1}} f_{s_{L-1}}\left(y_{(l+1) L-1}\right) p_{s_{L-1} 1} \\
p_{s_{1}}^{*} f_{s_{1}}\left(y_{l L+1}\right) p_{s_{2}}^{*} \cdots p_{s_{L-1}}^{*} f_{s_{L-1}}\left(y_{(l+1) L-1}\right) p_{1}^{*},
\end{array}
$$

and for $i=1,2, \ldots, L-1, p_{s_{i}}^{*} f_{s_{i}}\left(y_{l L+i}\right) \geq p_{h j} f_{j}\left(y_{l L+i}\right)$ for any $h, j \in S$.) Suppose $j \neq 1$ and $t_{1: L-1}$ realizes $p_{i j}^{(L-1)}\left(y_{l L}\right)$, i.e.

$$
\begin{aligned}
p_{i j}^{(L-1)}\left(y_{l L}\right)= & p_{i t_{1}} f_{t_{1}}\left(y_{l L+1}\right) p_{t_{1} t_{2}} \cdots \\
& \cdots p_{t_{L-2} t_{L-1}} f_{t_{L-1}}\left(y_{(l+1) L-1}\right) p_{t_{L-1} j} .
\end{aligned}
$$

Hence, with $t_{0}$ and $t_{L}$ standing for $i$ and $j$, respectively (and $s_{0}=s_{L}=1$ ), the left-hand side of (28) becomes

$$
\begin{aligned}
& \left(\frac{p_{t_{0} t_{1}} f_{t_{1}}\left(y_{l L+1}\right)}{p_{s_{0} s_{1}} f_{s_{1}}\left(y_{l L+1}\right)}\right)\left(\frac{p_{t_{1} t_{2}} f_{t_{2}}\left(y_{l L+2}\right)}{p_{s_{1} s_{2}} f_{s_{2}}\left(y_{l L+2}\right)}\right) \ldots \\
& \left(\frac{p_{t_{L-2}} t_{L-1} f_{t_{L-1}}\left(y_{(l+1) L-1}\right)}{p_{s_{L-2} s_{L-1}} f_{s_{L-1}}\left(y_{(l+1) L-1}\right)}\right)\left(\frac{p_{t_{L-1} t_{L}} f_{j}\left(y_{(l+1) L}\right)}{p_{s_{L-1} s_{L}} f_{1}\left(y_{(l+1) L}\right)}\right) .
\end{aligned}
$$

For $h=1, \ldots, L$ such that $t_{h} \neq s_{h}$,

$$
\frac{p_{t_{h-1} t_{h}} f_{t_{h}}\left(y_{l L+h}\right)}{p_{s_{h-1} s_{h}} f_{s_{h}}\left(y_{l L+h}\right)}<1-\epsilon \text {, since } y_{l L+h} \in \mathcal{X}_{s_{h}} \text {. }
$$

For all other $h, s_{h}=t_{h}$ and therefore, the left-hand side of (34) becomes $\frac{p_{t_{h-1} t_{h}}}{p_{s_{h-1} s_{h}}}=\frac{p_{t_{h-1} s_{h}}}{p_{s_{h}}^{*}} \leq 1$ (by property (14)). Since the last term of the product (33) above does satisfy (34) $(j \neq 1),(28)$ is thus proved. Suppose next that $t_{1}, \ldots, t_{L-1}$ realizes $p_{i 1}^{(L-1)}\left(y_{l L}\right)$. With $s_{0}=1$ and $t_{0}=i$, similarly to the previous arguments, we have

$$
\frac{p_{i 1}^{(L-1)}\left(y_{l L}\right)}{p_{11}^{(L-1)}\left(y_{l L}\right)}=\prod_{h=1}^{L-1}\left(\frac{p_{t_{h-1} t_{h}} f_{t_{h}}\left(y_{l L+h}\right)}{p_{s_{h-1} s_{h}} f_{s_{h}}\left(y_{l L+h}\right)}\right) \frac{p_{t_{L-1} 1}}{p_{s_{L-1} 1}} \leq 1,
$$

implying (27).

Let us now prove (29). To that end, note that for all states $h, i, j \in S$ such that $p_{j h}>0$, it follows from the definitions (7) and (17) that

$$
\frac{p_{i h}}{p_{j h}} \leq \frac{p_{h}^{*}}{p_{j h}} \leq A
$$

If $R=1$, then (29) becomes

$$
p_{i j} f_{j}\left(y_{0}\right) \leq A p_{b_{0} 1} f_{1}\left(y_{0}\right)
$$

By the definition of $\mathcal{X}_{1}$ (recall that $y_{0} \in \mathcal{X}_{1}$ ), we have that for every $i, j \in S p_{i j} f_{j}\left(y_{0}\right) \leq p_{1}^{*} f_{1}\left(y_{0}\right)$. Using (35) with $h=1$ and $j=b_{0}$, we get $p_{1}^{*} f_{1}\left(y_{0}\right) \leq A p_{b_{0} 1} f_{1}\left(y_{0}\right)\left(p_{b_{0} 1}>0\right.$ by the construction of $\mathbf{b} \S \mathrm{IV}-\mathrm{A} 3)$. Putting these all together, we obtain

$$
p_{i j} f_{j}\left(y_{0}\right)<p_{1}^{*} f_{1}\left(y_{0}\right) \leq A p_{b_{0} 1} f_{1}\left(y_{0}\right) \text {, as required. }
$$

Consider the case $R>1$. Let $t_{1: R-1}$ be a path that realizes $p_{i j}^{(R-1)}\left(z_{m}\right)$, i.e. $p_{i j}^{(R-1)}\left(z_{m}\right)=$

$$
p_{i t_{1}} f_{t_{1}}\left(y_{1}^{\prime}\right) p_{t_{1} t_{2}} f_{t_{2}}\left(y_{2}^{\prime}\right) \cdots p_{t_{R-2} t_{R-1}} f_{t_{R-1}}\left(y_{R-1}^{\prime}\right) p_{t_{R-1} j} .
$$

By the definition of $\mathcal{X}_{l}(\S \mathrm{IV}-\mathrm{A} 1)$ and the facts that $y_{r}^{\prime} \in \mathcal{X}_{b_{r}}$, $r=1,2, \ldots, R-1$, and $y_{0} \in \mathcal{X}_{1}$, we have

$$
\begin{aligned}
p_{i j}^{(R-1)}\left(z_{m}\right) f_{j}\left(y_{0}\right) \leq p_{b_{1}}^{*} f_{b_{1}}\left(y_{1}^{\prime}\right) p_{b_{2}}^{*} f_{b_{2}}\left(y_{2}^{\prime}\right) \cdots \\
p_{b_{R-1}}^{*} f_{b_{R-1}}\left(y_{R-1}^{\prime}\right) p_{1}^{*} f_{1}\left(y_{0}\right) .
\end{aligned}
$$


Now, by the construction of $\mathbf{b}(\S \mathrm{IV}-\mathrm{A} 3), p_{b_{r-1} b_{r}}>0$ for $r=$ $1, \ldots, R,\left(b_{R}=1\right)$. Thus, the argument behind (35) applies here to bound the right-hand side of (36) from above by

$$
\begin{aligned}
& A p_{b_{0} b_{1}} f_{b_{1}}\left(y_{1}^{\prime}\right) A p_{b_{1} b_{2}} f_{b_{2}}\left(y_{2}^{\prime}\right) \cdots \\
& A p_{b_{R-2} b_{R-1}} f_{b_{R-1}}\left(y_{R-1}^{\prime}\right) A p_{b_{R-1} 1} f_{1}\left(y_{0}\right)= \\
& A^{R} p_{b_{0} 1}^{(R-1)}\left(z_{m}\right) f_{1}\left(y_{0}\right), \text { as required. }
\end{aligned}
$$

Let us now prove (30). If $m=1$ then (30) becomes

$$
p_{i j}^{(P)}\left(y_{2 k L}\right) \leq p_{1 j}^{(P)}\left(y_{2 k L}\right) q^{-1} \quad \forall j \in C \forall i \in S .
$$

If $P=0$, then (37) reduces to $p_{i j} \leq p_{1 j} q^{-1}$ which is true, because in this case the state $q_{1}=q_{T}=1$ belongs to $C$ (§IV-A3) and $p_{1 j} q^{-1} \geq 1$ ((15), (16) with $m=1$ ). To see why (37) is true with $P \geq 1$, note that by the same argument as used for proving (27) and (28), we now get $\forall h, l \in S$

$$
p_{1 a_{P}}^{(P-1)}\left(y_{2 k L}\right) f_{a_{P}}\left(y_{P}^{\prime \prime}\right) \geq p_{h^{\prime}, l}^{(P-1)}\left(y_{2 k L}\right) f_{l}\left(y_{P}^{\prime \prime}\right) .
$$

Also, since $a_{P}=q_{1} \in C$ (§IV-A3), $p_{a_{P} j} q^{-1} \geq 1$ ((15), (16) with $m=1)$. Thus $p_{i j}^{(P)}\left(y_{2 k L}\right)=$

$$
\begin{aligned}
& \text { by }{ }^{(4)} \max _{l \in S} p_{i l}^{(P-1)}\left(y_{2 k L}\right) f_{l}\left(y_{P}^{\prime \prime}\right) p_{l j} \\
& \stackrel{\text { by }}{\leq} p_{1 a_{P}}^{(P-1)}\left(y_{2 k L}\right) f_{a_{P}}\left(y_{P}^{\prime \prime}\right) \max _{l \in S} p_{l j} \\
& \leq p_{1 a_{P}}^{(P-1)}\left(y_{2 k L}\right) f_{a_{P}}\left(y_{P}^{\prime \prime}\right) \\
& \leq p_{1 a_{P}}^{(P-1)}\left(y_{2 k L}\right) f_{a_{P}}\left(y_{P}^{\prime \prime}\right) p_{a_{P} j} q^{-1} \stackrel{\text { by }}{\leq} p_{1 j}^{(P)}\left(y_{2 k L}\right) q^{-1} \text {. }
\end{aligned}
$$

For $m>1$, let $t_{1: m-1}$ be a path realizing $p_{h j}^{(m-1)}\left(y_{P}^{\prime \prime}\right)$. Thus,

$$
\begin{aligned}
p_{h j}^{(m-1)} & \left(y_{P}^{\prime \prime}\right)= \\
& =p_{h t_{1}} f_{t_{1}}\left(z_{1}^{\prime}\right) p_{t_{1} t_{2}} f_{t_{2}}\left(z_{2}^{\prime}\right) \cdots f_{t_{m-1}}\left(z_{m-1}^{\prime}\right) p_{t_{m-1} j} \\
& <K^{m-1} .
\end{aligned}
$$

(This is true since $z_{r}^{\prime} \in \mathcal{Z}$ for $r=1,2, \ldots, m-1$ ( $(\mathrm{IV}-\mathrm{A} 2)$ and thus, for $p_{h j}^{(m-1)}\left(y_{P}^{\prime \prime}\right)$ to be positive it is necessary that $t_{r} \in C, r=1, \ldots, m-1$, implying $f_{t_{r}}\left(z_{r}^{\prime}\right)<K$.) Now, let $t_{1: m-1}$ realize $p_{a_{P} j}^{(m-1)}\left(y_{P}^{\prime \prime}\right)$, which is clearly positive, with $t_{r} \in C, r=1, \ldots, m-1\left(z_{r}^{\prime} \in \mathcal{Z}\right.$ for $r=1,2, \ldots, m-1$ ), and $a_{P}, j \in C$ (recall the positivity assumption on $\mathbb{Q}^{m}, \oint$ IV-A4). We thus have $p_{a_{P} j}^{(m-1)}\left(y_{P}^{\prime \prime}\right)=$ $p_{a_{P} t_{1}} f_{t_{1}}\left(z_{1}^{\prime}\right) p_{t_{1} t_{2}} f_{t_{2}}\left(z_{2}^{\prime}\right) \cdots f_{t_{m-1}}\left(z_{m-1}^{\prime}\right) p_{t_{m-1} j} \geq$

$$
\geq q_{a_{P} j}^{*} f_{t_{1}}\left(z_{1}^{\prime}\right) f_{t_{2}}\left(z_{2}^{\prime}\right) \cdots f_{t_{m-1}}\left(z_{m-1}^{\prime}\right)>q \delta^{m-1} .
$$

Combining the bounds of (39) and (40) $(q>0$, (16)), we obtain:

$$
p_{h j}^{(m-1)}\left(y_{P}^{\prime \prime}\right)<p_{a_{P} j}^{(m-1)}\left(y_{P}^{\prime \prime}\right)\left(\frac{K}{\delta}\right)^{m-1} / q
$$

Finally, $p_{i j}^{(P+m-1)}\left(y_{2 k L}\right)=$

$$
\begin{aligned}
& \stackrel{\text { by }}{=}{ }^{(4)} \max _{l \in S} p_{i l}^{(P-1)}\left(y_{2 k L}\right) f_{l}\left(y_{P}^{\prime \prime}\right) p_{l j}^{(m-1)}\left(y_{P}^{\prime \prime}\right) \\
& \text { by (38), (41) } p_{1 a_{P}}^{(P-1)}\left(y_{2 k L}\right) f_{a_{P}}\left(y_{P}^{\prime \prime}\right) p_{a_{P} j}^{(m-1)}\left(y_{P}^{\prime \prime}\right)\left(\frac{K}{\delta}\right)^{m-1} / q \\
& \stackrel{\text { by }}{\leq} p_{1 j}^{(P+m-1)}\left(y_{2 k L}\right)\left(\frac{K}{\delta}\right)^{m-1} / q \text {. }
\end{aligned}
$$

10) $\gamma_{j} \leq$ const $\times \gamma_{1}$ : Combining (24), (26), and (29), we see that for every state $j \in S$,

$$
\begin{aligned}
\gamma_{j} & \stackrel{\text { by }}{=}{ }^{(24)} \beta_{i_{\gamma}(j)} p_{i_{\gamma}(j) j}^{(R-1)}\left(z_{m}\right) f_{j}\left(y_{0}\right) \\
\text { by } & \quad{ }^{(29)} \beta_{i_{\gamma}(j)} p_{b_{0} 1}^{(R-1)}\left(z_{m}\right) f_{1}\left(y_{0}\right) A^{R} \\
& \text { by }{ }^{(26)} q^{-1}\left(\frac{K}{\delta}\right)^{m} A^{R} \beta_{b_{0}} p_{b_{0} 1}^{(R-1)}\left(z_{m}\right) f_{1}\left(y_{0}\right) \\
& \leq U \max _{i \in S} \beta_{i} p_{i 1}^{(R-1)}\left(z_{m}\right) f_{1}\left(y_{0}\right) \stackrel{\text { by } \stackrel{(24)}{=} U \gamma_{1},}{ }
\end{aligned}
$$

where

$$
U \stackrel{\text { def }}{=} q^{-1}\left(\frac{K}{\delta}\right)^{m} A^{R}
$$

Hence

$$
\gamma_{j} \leq U \gamma_{1} \quad \forall j \in S
$$

11) Further bounds on likelihoods: Let $l \geq 0$ and $n>$ 0 be integers such that $l+n \leq 2 k$ but arbitrary otherwise. Expanding $p_{11}^{(n L-1)}\left(y_{l L}\right)$ recursively according with (4), we obtain

$$
\begin{aligned}
& p_{11}^{(n L-1)}\left(y_{l L}\right)=\max _{i_{1: n-1} \in S^{n-1}} p_{1 i_{1}}^{(L-1)}\left(y_{l L}\right) f_{i_{1}}\left(y_{(l+1) L}\right) \times \\
& \times p_{i_{1} i_{2}}^{(L-1)}\left(y_{(l+1) L}\right) f_{i_{2}}\left(y_{(l+2) L}\right) \cdots p_{i_{n-2} i_{n-1}}^{(L-1)}\left(y_{(l+n-2) L}\right) \times \\
& \times \quad f_{i_{n-1}}\left(y_{(l+n-1) L}\right) p_{i_{n-1} 1}^{(L-1)}\left(y_{(l+n-1) L}\right) .
\end{aligned}
$$

Since for any $i_{1} \in S, p_{1 i_{1}}^{(L-1)}\left(y_{l L}\right) f_{i_{1}}\left(y_{(l+1) L}\right) \leq$ $p_{11}^{(L-1)}\left(y_{l L}\right) f_{1}\left(y_{(l+1) L}\right)$, as well as

$$
\begin{aligned}
p_{i_{r-1} i_{r}}^{(L-1)}\left(y_{(l+r-1) L}\right) f_{i_{r}}\left(y_{(l+r) L}\right) & \text { by } \stackrel{(28)}{\leq} \\
& p_{11}^{(L-1)}\left(y_{(l+r-1) L}\right) f_{1}\left(y_{(l+r) L}\right), r=2, \ldots, n-1,
\end{aligned}
$$

and since for any $i_{n-1} \in S$

$$
p_{i_{n-1} 1}^{(L-1)}\left(y_{(l+n-1) L}\right) \stackrel{\text { by }(27)}{\leq} p_{11}^{(L-1)}\left(y_{(l+n-1) L}\right),
$$

maximization (44) above is achieved as

$$
\begin{aligned}
& \text { follows: } p_{11}^{(n L-1)}\left(y_{l L}\right)= \\
& p_{11}^{(L-1)}\left(y_{l L}\right) f_{1}\left(y_{(l+1) L}\right) p_{11}^{(L-1)}\left(y_{(l+1) L}\right) f_{1}\left(y_{(l+2) L}\right) \cdots \\
& \cdots p_{11}^{(L-1)}\left(y_{(l+n-2) L}\right) f_{1}\left(y_{(l+n-1) L}\right) p_{11}^{(L-1)}\left(y_{(l+n-1) L}\right) .
\end{aligned}
$$

Now, we replace state 1 by generic states $i, j \in S$ on the both ends of the paths in (44) and repeat the above arguments. Thus, also using (45), we arrive at bound (46) below:

$$
\begin{gathered}
p_{i j}^{(n L-1)}\left(y_{l L}\right) f_{j}\left(y_{(l+n) L}\right) \leq \\
\prod_{u=l+1}^{l+n} p_{11}^{(L-1)}\left(y_{(u-1) L}\right) f_{1}\left(y_{u L}\right) \stackrel{\text { by }}{=}(45) \\
p_{11}^{(n L-1)}\left(y_{l L}\right) f_{1}\left(y_{(l+n) L}\right) \quad \forall i, j \in S .
\end{gathered}
$$

In particular, (46) states $\forall i, j \in S$

$$
p_{i j}^{(k L-1)}\left(y_{0}\right) f_{j}\left(y_{k L}\right) \leq p_{11}^{(k L-1)}\left(y_{0}\right) f_{1}\left(y_{k L}\right) .
$$


12) $\eta_{j} \leq$ const $\times \eta_{1}:$ In order to see

$$
\eta_{j} \leq U \eta_{1} \quad \forall j \in S
$$

$$
\begin{aligned}
& \text { note: } \eta_{j} \stackrel{(25)}{=} \max _{i \in S} \gamma_{i} p_{i j}^{(k L-1)}\left(y_{0}\right) f_{j}\left(y_{k L}\right) \\
& \stackrel{\text { by }}{\leq} \max _{i \in S} \gamma_{i} p_{11}^{(k L-1)}\left(y_{0}\right) f_{1}\left(y_{k L}\right) \stackrel{\text { by }}{\leq}
\end{aligned}
$$

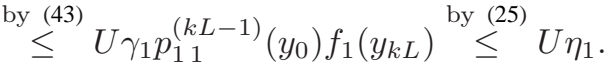

13) A representation of $\eta_{1}$ : Recall that $k$, the number of cycles in the $s$-path, was chosen sufficiently large for (18) to hold (in particular, $k>1$ ). We now prove that there exists $\kappa \in\{1, \ldots, k-1\}$ such that

$$
\eta_{1}=\delta_{1}\left(y_{\kappa L}\right) p_{1}^{((k-\kappa) L-1)}\left(y_{\kappa L}\right) f_{1}\left(y_{k L}\right) .
$$

The relation (49) states that (given observations $x_{1: u}$ ) a maximum-likelihood path (from time 1 , observation $x_{1}$ ) to time $u-m-P-k L$ (observation $y_{k L}$ ) goes through state 1 at time $u-m-P-2 k L+\kappa L$, that is when $y_{\kappa L}$ is observed.

To see this, suppose no such $\kappa$ existed.Then, applying (4) to (25) and recalling that $\delta_{1}\left(y_{\kappa L}\right)$ is introduced in (21), we would have

$$
\begin{aligned}
\eta_{1}= & \gamma_{j_{\eta}(1)} p_{j_{\eta}(1) j_{1}}^{(L-1)}\left(y_{0}\right) f_{j_{1}}\left(y_{L}\right) p_{j_{1} j_{2}}^{(L-1)}\left(y_{L}\right) \times \\
& \times f_{j_{2}}\left(y_{2 L}\right) p_{j_{2} j_{3}}^{(L-1)}\left(y_{2 L}\right) \cdots p_{j_{k-1} 1}^{(L-1)}\left(y_{(k-1) L}\right) f_{1}\left(y_{k L}\right)
\end{aligned}
$$

for some $j_{1} \neq 1, \ldots, j_{k-1} \neq 1$. Furthermore, this would imply $\eta_{1}<$

$$
\begin{aligned}
& \text { by } \stackrel{(28),(27)}{<} \gamma_{j_{\eta}(1)}(1-\epsilon)^{k-1} \prod_{i=1}^{k} p_{11}^{(L-1)}\left(y_{(i-1) L}\right) f_{1}\left(y_{i L}\right)
\end{aligned}
$$

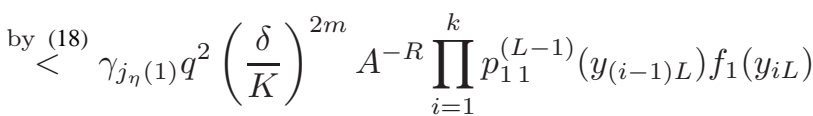

$$
\begin{aligned}
& \stackrel{\text { by }}{\leq}{ }^{(43)} \gamma_{1} U q^{2}\left(\frac{\delta}{K}\right)^{2 m} A^{-R} \prod_{i=1}^{k} p_{11}^{(L-1)}\left(y_{(i-1) L}\right) f_{1}\left(y_{i L}\right)
\end{aligned}
$$

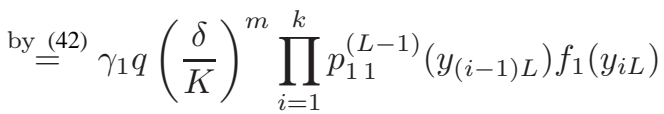

$$
\begin{aligned}
& <\gamma_{1} \prod_{i=1}^{k} p_{11}^{(L-1)}\left(y_{(i-1) L}\right) f_{1}\left(y_{i L}\right) .
\end{aligned}
$$

(The last inequality follows from $q \leq 1$ (16) and $\delta<K$, §IV-A2.) On the other hand, by definition (25) (and $k-1$-fold application of (4)), $\eta_{1} \geq \gamma_{1} \prod_{i=1}^{k} p_{11}^{(L-1)}\left(y_{(i-1) L}\right) f_{1}\left(y_{i L}\right)$, which evidently contradicts (50) above. Therefore, $\kappa$ satisfying (49) and $1 \leq \kappa<k$, does exist.

14) An implication of (45) and (49) for $\delta_{1}\left(y_{l L}\right)$ : Clearly, the arguments of the previous section (§IV-A13) are valid if $k$ is replaced by any $l \in\{k, \ldots, 2 k\}$. Hence the following generalization of (49): For some $\kappa(l)<l$

$$
\delta_{1}\left(y_{l L}\right)=\delta_{1}\left(y_{\kappa(l) L}\right) p_{11}^{((l-\kappa(l)) L-1)}\left(y_{\kappa(l) L}\right) f_{1}\left(y_{l L}\right) .
$$

We apply (51) recursively, starting with $\kappa^{(0)} \stackrel{\text { def }}{=} l$ and returning $\kappa^{(1)} \stackrel{\text { def }}{=} \kappa(l)<l$. If $\kappa^{(1)} \leq k$, we stop, otherwise we substitute $\kappa^{(1)}$ for $l$, and obtain $\kappa^{(2)} \stackrel{\text { def }}{=} \kappa(l)<\kappa^{(1)}$, and so, on until $\kappa^{(j)} \leq k$ for some $j>0$. Thus, $\delta_{1}\left(y_{l L}\right)=$

$$
\begin{array}{r}
=\delta_{1}\left(y_{\kappa^{(j)} L}\right) p_{11}^{\left(\left(\kappa^{(j-1)}-\kappa^{(j)}\right) L-1\right)}\left(y_{\kappa^{(j)} L}\right) f_{1}\left(y_{\kappa^{(j-1)} L}\right) \cdots \\
p_{11}^{\left(\left(l-\kappa^{(1)}\right) L-1\right)}\left(y_{\kappa^{(1)} L}\right) f_{1}\left(y_{l L}\right) .
\end{array}
$$

Applying (45) to the appropriate factors of the right-hand side of (52) above, we obtain:

$$
\begin{gathered}
\delta_{1}\left(y_{l L}\right)=\delta_{1}\left(y_{\kappa^{(j)} L}\right) p_{11}^{(L-1)}\left(y_{\kappa^{(j)} L}\right) f_{1}\left(y_{\left(\kappa^{(j)}+1\right) L}\right) \cdots \\
p_{11}^{(L-1)}\left(y_{(k-1) L}\right) f_{1}\left(y_{k L}\right) \cdots p_{11}^{(L-1)}\left(y_{k L}\right) f_{1}\left(y_{(k+1) L}\right) \cdots \\
p_{11}^{(L-1)}\left(y_{\left(\kappa^{(j-1)}-1\right) L}\right) f_{1}\left(y_{\kappa^{(j-1)} L}\right) \cdots \\
p_{11}^{(L-1)}\left(y_{\left(\kappa^{(1)}-1\right) L}\right) f_{1}\left(y_{\kappa^{(1)} L}\right) \cdots \\
p_{11}^{(L-1)}\left(y_{(l-1) L}\right) f_{1}\left(y_{l L}\right) .
\end{gathered}
$$

Also, according to (45),

$$
\begin{aligned}
& \delta_{1}\left(y_{\kappa^{(j)} L}\right) p_{11}^{(L-1)}\left(y_{\kappa^{(j)} L}\right) f_{1}\left(y_{\left(\kappa^{(j)}+1\right) L}\right) \cdots \\
& p_{11}^{(L-1)}\left(y_{(k-1) L}\right)=\delta_{1}\left(y_{\kappa^{(j)} L}\right) p_{11}^{\left(\left(k-\kappa^{(j)}\right) L-1\right)}\left(y_{\kappa^{(j)} L}\right) .
\end{aligned}
$$

At the same time,

$$
\delta_{1}\left(y_{\kappa^{(j)} L}\right) p_{11}^{\left(\left(k-\kappa^{(j)}\right) L-1\right)}\left(y_{\kappa^{(j)} L}\right) f_{1}\left(y_{k L}\right) \stackrel{\text { by }}{\leq}{ }^{(4)} \eta_{1} .
$$

However, we cannot have the strict inequality in (54) above since that, by virtue of (53), would contradict maximality of $\delta_{1}\left(y_{l L}\right)$. We have thus arrived at $\delta_{1}\left(y_{l L}\right)=$ $\eta_{1} p_{11}^{(L-1)}\left(y_{k L}\right) f_{1}\left(y_{(k+1) L}\right) \cdots$

$$
\cdots p_{11}^{(L-1)}\left(y_{(l-1) L}\right) f_{1}\left(y_{l L}\right) .
$$

In summary, for any $l \geq k$ and $l \leq 2 k$ there exists a realization of $\delta_{1}\left(y_{l L}\right)$ that goes through state 1 every time when $y_{i L}, i=k, \ldots, l$, is observed.

15) $y_{k L}$ is a $(k L+m+P)$-order 1-node: In $\S \mathrm{IV}$-A16, we will prove that for any $i \in S, i \neq 1$, and any $j \in C$,

$$
\eta_{i} p_{i j}^{(k L+m+P-1)}\left(y_{k L}\right) \leq \eta_{1} p_{1 j}^{(k L+m+P-1)}\left(y_{k L}\right),
$$

which implies that $y_{k L}$ is a 1-node of order $k L+m+P$. Indeed, let $l \in S$ be arbitrary. Since $f_{j}\left(z_{m}^{\prime}\right)=0$ for every $j \in S \backslash C$, any maximum likelihood path to state $l$ at time $u+1$ (observation $x_{u+1}$ ) must go through a state in $C$ at time $u$ (observation $x_{u}=z_{m}^{\prime}$.) Formally,

$$
\begin{aligned}
& \eta_{i} p_{i l}^{(k L+m+P)}\left(y_{k L}\right)= \\
& \quad= \max _{j \in S} \eta_{i} p_{i j}^{(k L+m+P-1)}\left(y_{k L}\right) f_{j}\left(z_{m}^{\prime}\right) p_{j l} \\
&= \max _{j \in C} \eta_{i} p_{i j}^{(k L+m+P-1)}\left(y_{k L}\right) f_{j}\left(z_{m}^{\prime}\right) p_{j l} \\
& \stackrel{\text { by (56) }}{\leq} \max _{j \in C} \eta_{1} p_{1 j}^{(k L+m+P-1)}\left(y_{k L}\right) f_{j}\left(z_{m}^{\prime}\right) p_{j l} \\
& \stackrel{\text { by (4) }}{=} \eta_{1} p_{1 l}^{(k L+m+P)}\left(y_{k L}\right) .
\end{aligned}
$$

Therefore, by Definition $2.1 y_{k L}$ is a 1-node of order $k L+$ $m+P$. 
16) Proof of (56): Let $i \in S$ and $j \in C$ be arbitrary. Let state $j^{*} \in S$ be such that $p_{i j}^{(k L+m+P-1)}\left(y_{k L}\right)=$

$$
p_{i j^{*}}^{(k L-1)}\left(y_{k L}\right) f_{j^{*}}\left(y_{2 k L}\right) p_{j^{*} j}^{(m+P-1)}\left(y_{2 k L}\right)=
$$

$\nu\left(i, j^{*}\right) p_{j^{*} j}^{(m+P-1)}\left(y_{2 k L}\right)$, where

$$
\nu(i, j) \stackrel{\text { def }}{=} p_{i j}^{(k L-1)}\left(y_{k L}\right) f_{j}\left(y_{2 k L}\right), \quad \text { for all } i, j \in S .
$$

We consider the following two cases separately:

1. There exists a path realizing $p_{i j^{*}}^{(k L-1)}\left(y_{k L}\right)$ and going through state 1 at the time of observing $y_{l L}$ for some $l \in\{k, \ldots, 2 k\} \cdot p_{i j^{*}}^{(k L-1)}\left(y_{k L}\right)=$

$$
p_{i 1}^{((l-k) L-1)}\left(y_{k L}\right) f_{1}\left(y_{l L}\right) p_{1 j^{*}}^{((2 k-l) L-1)}\left(y_{l L}\right) .
$$

Equation (57) above together with the fundamental recursion (4) yields the following:

$$
\begin{aligned}
& \eta_{i} p_{i j^{*}}^{(k L-1)}\left(y_{k L}\right)=
\end{aligned}
$$

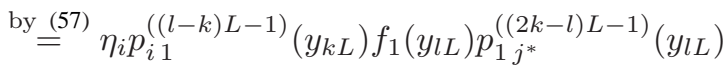

$$
\begin{aligned}
& \text { by (21), (4) } \delta_{1}\left(y_{l L}\right) p_{1 j}^{((2 k-l) L-1)}\left(y_{l L}\right) \text {. }
\end{aligned}
$$

At the same time, the right hand-side of (58) can be expressed as follows:

$$
\begin{aligned}
& \delta_{1}\left(y_{l L}\right) p_{1 j^{*}}^{((2 k-l) L-1)}\left(y_{l L}\right)
\end{aligned}
$$

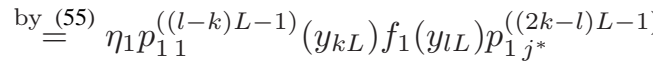

$$
\begin{aligned}
& \text { by } \stackrel{(45)}{=} \eta_{1} p_{1 j^{*}}^{(k L-1)}\left(y_{k L}\right) \text {. }
\end{aligned}
$$

Therefore, if there exists $l \in\{k, \ldots, 2 k\}$ such that (57) holds, we have by virtue of (58) and (59): $\eta_{i} p_{i j^{*}}^{(k L-1)}\left(y_{k L}\right) \leq \eta_{1} p_{1 j^{*}}^{(k L-1)}\left(y_{k L}\right)$, that is

$$
\eta_{i} \nu\left(i, j^{*}\right) \leq \eta_{1} \nu\left(1, j^{*}\right) .
$$

$$
\begin{aligned}
\text { Hence, } & \eta_{i} p_{i j}^{(k L+m+P-1)}\left(y_{k L}\right)= \\
\text { by (57) } & \eta_{i} \nu\left(i, j^{*}\right) p_{j^{*} l}^{(m+P-1)}\left(y_{2 k L}\right) \\
\stackrel{\text { by (60) }}{\leq} & \eta_{1} \nu\left(1, j^{*}\right) p_{j^{*} j}^{(m+P-1)}\left(y_{2 k L}\right) \\
\stackrel{\text { by (4) }}{\leq} & \eta_{1} p_{1 j}^{(k L+m+P-1)}\left(y_{k L}\right)
\end{aligned}
$$

and (56) holds.

2. Assume now that no path exists to satisfy (57). Argue as for (50) to obtain $\nu\left(i, j^{*}\right)<$

$$
(1-\epsilon)^{k-1} \prod_{n=k+1}^{2 k} p_{11}^{(L-1)}\left(y_{(n-1) L}\right) f_{1}\left(y_{n L}\right) .
$$

By 45, the (partial likelihood) product in the right-hand side of (61) equals $\nu(1,1)$. Thus,

$$
\begin{aligned}
& \eta_{i} \nu\left(i, j^{*}\right) p_{j^{*} j}^{(m+P-1)}\left(y_{2 k L}\right)<
\end{aligned}
$$

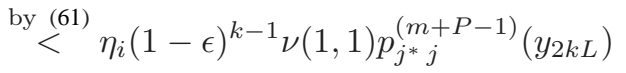

$$
\begin{aligned}
& \stackrel{\text { by }}{<}{ }^{(18)} \eta_{i} q^{2}\left(\frac{\delta}{K}\right)^{2 m} A^{-R} \nu(1,1) p_{j^{*} j}^{(m+P-1)}\left(y_{2 k L}\right) \\
& \text { by (42), (48) } \eta_{1} q\left(\frac{\delta}{K}\right)^{m} \nu(1,1) p_{j^{*} j}^{(m+P-1)}\left(y_{2 k L}\right) \text {. }
\end{aligned}
$$

Clearly, the actual probability of observing $q_{1: M}$ is positive, as required. By the constructions of $\S \S I V-A 1-I V-A 3$, the conditional probability of $B$ below, given $q_{1: M}$, is evidently positive, as required.

$$
\begin{aligned}
B \stackrel{\text { def }}{=} \mathcal{Z}^{m+1} & \times \mathcal{X}_{b_{1}} \times \cdots \times \mathcal{X}_{b_{R-1}} \times \mathcal{X}_{1} \times \mathcal{X}_{s_{1}} \times \\
\cdots & \times \mathcal{X}_{s_{2 k L-1}} \times \mathcal{X}_{1} \times \mathcal{X}_{a_{1}} \times \cdots \times \mathcal{X}_{a_{P}} \times \mathcal{Z}^{m}
\end{aligned}
$$

Finally, since the sequence (20) below was chosen from $B$ arbitrarily (§IV-A6) and has been shown to be an $l$-barrier of order $r$, this completes the proof of the Lemma.

$$
\left(z_{0: m}, y_{1: R-1}^{\prime}, y_{0: 2 L k}, y_{1: P}^{\prime \prime}, z_{1: m}^{\prime}\right) \in B .
$$

\section{B. Proof of Lemma 3.2}

Proof: We use the notation of the previous proof in $\S I V-A$ and consider the following two distinct situations: First (§IV-B1), all barriers from $B$ as constructed in the proof of Lemma 3.2 are already separated. Obviously, there is nothing to do in this case. The second situation (§IV-B2) is complementary, in which case a simple extension will immediately ensure separation.

1) All $y \in B$ are already separated: Recall the definition of $\mathcal{Z}$ from $\S I V-A 2$. Consider the two cases in the definition separately. First, suppose $\mathcal{Z}=\hat{\mathcal{Z}} \backslash\left(\cup_{l \in S} \mathcal{X}_{l}\right)$, in which case $\mathcal{Z}$ and $\mathcal{X}_{l}$ are disjoint for every $l \in S$. This implies that every barrier (20) is already separated. Indeed, for any $w, 1 \leq w \leq$ $r$, and for any $y \in B$, the fact that $y_{M-\max (m, w)} \notin \mathcal{Z}$, for example, makes it impossible for $\left(y_{1: w}^{\prime}, y_{1: M-w}\right) \in B$ for any 
$y_{1: w}^{\prime} \in \mathcal{X}^{w}$. Consider now the case when $\mathcal{Z}=\hat{\mathcal{Z}} \cap \mathcal{X}_{s}$ for some $s \in C$. Then

$$
\begin{array}{r}
B \subset \mathcal{X}_{s}^{m+1} \times \mathcal{X}_{b_{1}} \times \cdots \times \mathcal{X}_{b_{R-1}} \times \mathcal{X}_{1} \times \mathcal{X}_{s_{1}} \times \cdots \\
\mathcal{X}_{s_{2 k L-1}} \times \mathcal{X}_{1} \times \mathcal{X}_{a_{1}} \times \cdots \times \mathcal{X}_{a_{P-1}} \times \mathcal{X}_{s}^{m+1}
\end{array}
$$

Let $y \in B$ be arbitrary. Assume first $L>1$. By construction ( $\left(\mathrm{IV}-\mathrm{A} 3\right.$ ), the states $s_{1}, \ldots, s_{L}$ are all distinct. We now show that $\left(y_{1: w}^{\prime}, y_{1: M-w}\right) \notin B$ for any $y_{1: w}^{\prime} \in \mathcal{X}^{w}$ when $1 \leq w \leq r$. Note that the sequence

$$
q_{m+2: m+R+2 k L+P+1}=\left(b_{1: R-1}, 1, s_{1: 2 k L-1}, 1, a_{1: P-1}, s\right)
$$

is such that no two consecutive states are equal. It is straightforward to verify that there exist indices $j, 0 \leq j \leq m-1$, such that, when shifted $w$ positions to the right, the pair $y_{j+1 j+2} \in \mathcal{X}_{s}^{2}$ would at the same time have to belong to $\mathcal{X}_{q_{j+1+w}} \times \mathcal{X}_{q_{j+2+w}}$ with $m+1 \leq j+1+w<j+2+w \leq$ $m+R+2 k L+1+P$. This is clearly a contradiction since $\mathcal{X}_{q_{j+1+w}}$ and $\mathcal{X}_{q_{j+2+w}}$ are disjoint for that range of indices $j$. A verification of the above fact simply amounts to verifying that the inequality $\max (0, m-w) \leq j \leq \min (m-1, m+R+$ $2 k L-1+P-w)$ is consistent for any $w$ from the admissible range:

i.) When $0 \geq m-w, m-1 \leq m+R+2 k L-1+P-w$ $(m \leq w \leq \min (r, R+2 k L+P)), 0 \leq j \leq m-1$ is evidently consistent.

ii.) When $0 \geq m-w, m-1>m+R+2 k L-1+P-w$ $(\max (m, R+2 k L+P) \leq w \leq r), 0 \leq j \leq m+R+$ $2 k L-1+P-w$ is also consistent since $m+R+2 k L-$ $1+P-r=R+k L-1 \geq 0$.

iii.) When $0<m-w, m-1 \leq m+R+2 k L-1+P-w$ $(1 \leq w \leq \min (m-1, R+2 k L+P)), m-w \leq j \leq m-1$ is consistent since $w \geq 1$.

iv.) When $0<m-w, m-1>m+R+2 k L-1+P-w$ $(\max (1, R+2 k L+P-1) \leq w<m), m-w \leq j \leq m+$ $R+2 k L-1+P-w$ is consistent since $R+2 k L-1 \geq 0$.

Next consider the case of $L=1$ but $s \neq 1$ (that is, $P>0$ ). Then $B \subset \mathcal{X}_{s}^{m+1} \times \mathcal{X}_{b_{1}} \times \cdots$

$$
\times \mathcal{X}_{b_{R-1}} \times \mathcal{X}_{1}^{2 k+1} \times \mathcal{X}_{a_{1}} \times \cdots \times \mathcal{X}_{a_{P-1}} \times \mathcal{X}_{s}^{m+1} .
$$

If $s \neq 1$, then also $b_{i} \neq 1, i=1, \ldots, R-1$ and $a_{i} \neq 1$, $i=1, \ldots, P-1$. To see that $y$ is separated in this case, simply note that $y_{M-\max (w, m+1)} \notin \mathcal{X}_{s}$ for any admissible $w$.

2) Barriers $y \in B$ need not be separated: Finally, we consider the case when $L=1$ and $s=1$ (where $s \in C$ is such that $\mathcal{Z}=\hat{\mathcal{Z}} \cap \mathcal{X}_{s}$ ). This implies that $P=0,1 \in C$, and $p_{11}>0$, which in turn implies that $R=1$, and

$$
B \subset \mathcal{X}_{1}^{m+1} \times \mathcal{X}_{1}^{2 k+1} \times \mathcal{X}_{1}^{m+1}=\mathcal{X}_{1}^{2 m+2 k+3} .
$$

Clearly, the barriers from $B$ need not be, and indeed, are not separated. It is, however, easy to extend them to separated ones. Indeed, let $q_{0} \neq 1$ be such that $p_{q_{0} 1}>0$ and redefine $B \stackrel{\text { def }}{=} \mathcal{X}_{q_{0}} \times B$. Evidently, any shift of any $y \in B$ by $w$ $(1 \leq w \leq r)$ positions to the right makes it impossible for $y_{1}$ to be simultaneously in $\mathcal{X}_{q_{0}}$ and in $\mathcal{X}_{1}$ (since the latter sets are disjoint, $\S I V-A 1$ ).

\section{CONCLUSION}

As discussed in $\S \mathrm{I}$ and $\S \mathrm{I}-\mathrm{A}$ in particular, the proper infinite alignments ( $(I I-B)$ allow us to define the decoding process $V$ which is regenerative and can further be stationarized to become ergodic [7]. This in turn allows us to study the distribution and asymptotic properties not only of the Viterbi process $V$ but also of the joint process $(X, V)$. In particular, this reveals how different these properties are from the properties of the underlying chain $Y$ and $\operatorname{HMM}(X, Y)$, respectively. More specifically, since the process $V$ (resp. $(X, V)$ ) can deviate from the process $Y$ (resp. $(X, Y)$ ) significantly, using the Viterbi alignments $v_{1: n}$ as estimates for the hidden paths $Y_{1: n}$ might lead to incorrect conclusions not only for finite $n$ (as generally appreciated) but also in the limit as $n \rightarrow \infty$ [7].

This certainly does not mean that one should not make inference based on $V$ but simply suggests that the aforementioned differences may need to be taken into account. One example of how these asymptotic differences can be successfully accounted for is the adjusted Viterbi training for HMM parameter estimation [11], [12], [7].

If known - possibly estimated - these differences might also be appreciated when the Viterbi paths are used for prediction, or segmentation, of $Y$, e.g. in speech segmentation or in segmentation of DNA sequences into coding and non-coding regions, or in detection of $\mathrm{CpG}$ islands in DNA sequences [15]. Indeed, in segmentation of DNA sequences, the underlying chain $Y$ has few, often two, states (e.g. coding and noncoding regions, or $\mathrm{CpG}$ islands and non- $\mathrm{CpG}$ regions), the probabilities of transitions between the states are very low, hence the true $(Y)$ and predicted $(V)$ hidden paths consist of long constant blocks. At the same time, it has been noted that the predicted constant blocks can be somewhat longer than what the chain parameters would suggest. With the help of the infinite Viterbi process $V$ it is now clear that this discrepancy is not simply due to the random fluctuations but is systematic, does not vanish asymptotically, and is a direct consequence of that the transition probabilities of $V$ do indeed often underestimate the true ones. Note that in these examples, unlike in the estimation of the HMM emission parameters, the overall performance is directly linked to the accuracy of the transition probability estimates. Thus, finding the differences between the processes $(X, Y)$ and $(X, V)$ in this case might help find better alignments.

\section{ACKNOWLEDGMENT}

The first author has been supported by the Estonian Science Foundation Grant 7553. The authors thank Eurandom (The Netherlands) for initiating and stimulating their research on hidden Markov models, of which this work has been an integral part. The authors also thank Dr. A. Caliebe for valuable discussions and for emphasizing the significance of the topic of path estimation in HMMs.

\section{REFERENCES}

[1] Y. Ephraim and N. Merhav, "Hidden Markov processes," IEEE Trans. Inform. Theory, vol. 48, no. 6, pp. 1518-1569, 2002, special issue on Shannon theory: perspective, trends, and applications. 
[2] V. Genon-Catalot, T. Jeantheau, and C. Larédo, "Stochastic volatility models as hidden Markov models and statistical applications," Bernoulli, vol. 6, no. 6, pp. 1051-1079, 2000.

[3] B. G. Leroux, "Maximum-likelihood estimation for hidden Markov models," Stochastic Process. Appl., vol. 40, no. 1, pp. 127-143, 1992.

[4] A. Viterbi, "Error bounds for convolutional codes and an asymptotically optimum decoding algorithm," IEEE Trans. Inform. Theory, vol. 13, no. 2, pp. 260-269, 1967.

[5] L. Rabiner, "A tutorial on hidden Markov models and selected applications in speech recognition," Proc. IEEE, vol. 77, no. 2, pp. 257-286, 1989.

[6] O. Cappé, E. Moulines, and T. Rydén, Inference in hidden Markov models, ser. Springer Series in Statistics. New York: Springer, 2005, with Randal Douc's contributions to Chapter 9 and Christian P. Robert's to Chapters 6, 7 and 13, With Chapter 14 by Gersende Fort, Philippe Soulier and Moulines, and Chapter 15 by Stéphane Boucheron and Elisabeth Gassiat.

[7] J. Lember and A. Koloydenko, "The Adjusted Viterbi training for hidden Markov models," Bernoulli, vol. 14, no. 1, pp. 180-206, 2008.

[8] A. Caliebe and U. Rösler, "Convergence of the maximum a posteriori path estimator in hidden Markov models," IEEE Trans. Inform. Theory, vol. 48 , no. 7, pp. 1750-1758, 2002.

[9] A. Caliebe, "Properties of the maximum a posteriori path estimator in hidden Markov models," IEEE Trans. Inform. Theory, vol. 52, no. 1, pp. 41-51, 2006.

[10] J. A. Kogan, "Hidden Markov models estimation via the most informative stopping times for the Viterbi algorithm," in Image models (and their speech model cousins) (Minneapolis, MN, 1993/1994), ser. IMA Vol. Math. Appl. New York: Springer, 1996, vol. 80, pp. 115-130.

[11] J. Lember and A. Koloydenko, "Adjusted Viterbi training: A proof of concept," Probab. Eng. Inf. Sci., vol. 21, no. 3, pp. 451-475, 2007.

[12] A. Koloydenko, M. Käärik, and J. Lember, "On adjusted Viterbi training," Acta Appl. Math., vol. 96, no. 1-3, pp. 309-326, 2007.

[13] F. Jelinek, "Continuous speech recognition by statistical methods," Proc. IEEE, vol. 64, pp. 532-556, 1976.

[14] J. Lember and A. Koloydenko, "Infinite Viterbi alignments in the twostate hidden Markov models," in Proc. 8th Tartu Conf. Multivariate Statist., July 2007, accepted.

[15] R. Durbin, S. Eddy, K. A., and G. Mitchison, Biological Sequence Analysis: Probabilistic Models of Proteins and Nucleic Acids. Cambridge University Press, 1998.

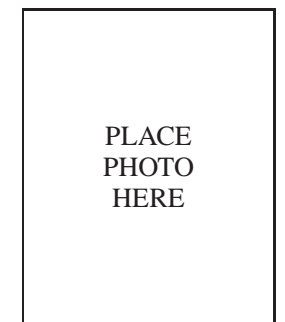

Alexey Koloydenko received the B.S. degrees in physics and mathematics (with information systems minor) in 1994 from the Voronezh University, Russian Federation and Norwich University, USA, respectively. He received in 1996 the M.S.(tech.) degree in physics and radio-electronics from the Voronezh University, Russian Federation, and the M.S. degree in mathematics and statistics from the University of Massachusetts at Amherst, USA. He received the Ph.D. degree in mathematics and statistics from the University of Massachusetts at

Amherst, USA, in 2000.

He held Postdoctoral Research and Teaching positions with the Department of Mathematics and Statistics of the University of Massachusetts at Amherst, Statistics and Computer Science Departments of the University of Chicago, and Eurandom, The Netherlands, in 2000, 2001-2002, and 2002-2005, respectively. Since 2005 he has been a Lecturer in Statistics at the University of Nottingham, UK. His research interests include statistical processing and analysis of images, diffusion weighted MRI, algebraic aspects of probability theory and statistics, and hidden Markov models.

Dr. Koloydenko has been a member of the Pattern Analysis, Statistical Modelling and Computational Learning European network (PASCAL) since 2004.

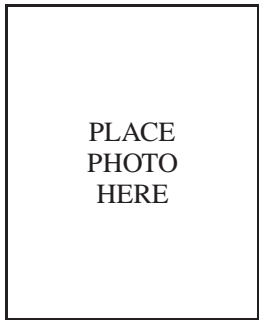

Jüri Lember was born in 1968 in Tallinn, Estonia. He received the diploma and M.Sc. degrees in mathematical statistics in 1992 and 1994, respectively, from the University of Tartu, Estonia. He received the Ph.D. degree in mathematics from the University of Tartu, Estonia, in 1999.

He completed his compulsory military service in 1987-1989, and was a Postdoctoral Research Fellow in the Institute of Mathematical Statistics, University of Tartu, in 1999-2000. He held a Postdoctoral Research position in Eurandom, The Netherlands, in 2001-2003. Since 2003, he has been a Lecture and a Senior Researcher in the Institute of Mathematical Statistics, University of Tartu. His scientific interests include probability theory, theoretical statistics, information theory, and speech recognition.

Dr. Lember has been a member of the Estonian statistical society as well as Estonian mathematical society since 2003. He has been awarded Estonian Science foundation grants for periods of 2004-2007 and 2008-2011. 